\title{
ON SMOOTHING COMPACT MEASURE SPACES BY MULTIPLICATION
}

\author{
BY \\ DOROTHY MAHARAM( $\left.{ }^{1}\right)$
}

\begin{abstract}
Let $\mu$ be a regular Borel probability measure on a compact Hausdorff space $S$, and let $m$ be Lebesgue measure on the unit interval $I$. It is proved that the measure-theoretic product $(S, \mu) \times\left(I^{w}, m^{w}\right)$, where $w$ is a large enough cardinal and $m^{w}$ denotes product Lebesgue measure, is "pseudoisometric" to $\left(I^{w}, m^{w}\right)$. Here a pseudo-isometry $\phi$ is a point-isometry except that, instead of $\phi(A)$ being measurable for every measurable $A$, it is required only that $A$ differ by a null set from a set with measurable image. If instead $\mu$ is a Baire probability measure and $S$ is a Baire subset of $I^{w}$, then $(S, \mu) \times\left(I^{w}, m^{w}\right)$ is point-isometric to $\left(I^{w}, m^{w}\right)$. Finally it is shown that (roughly speaking) continuous maps can be "smoothed" into projection maps (to within pseudo-isometries) by multiplication by suitable projection maps.
\end{abstract}

1. Introduction. The measure space $\left(I^{w}, m^{w}\right)$ (that is, the product, with product measure $m^{w}$, of $w$ copies of the unit interval $I$, each with Lebesgue measure $m ; w$ is an arbitrary cardinal) has many desirable properties; for instance, its measure is completion regular $[1$, p. 230], $[4$, p. 993], it has the strong lifting property [8], it has "sufficiently many measure-preserving transformations" [5], and in fact every endomorphism of its measure algebra can be realized by a surjective point-mapping (as the author expects to show elsewhere). $\left({ }^{2}\right)$ One would like to assert that an arbitary compact Hausdorff space $S$ with a "nice" measure $\mu$ (say a regular Borel measure with $\mu(S)=1$ ) has similar properties; but this is unfortunately not the case, in general. $\left({ }^{3}\right)$ This suggests that

Presented to the Society, June 25, 1971 under the title An isomorphism theorem for compact measure spaces; received by the editors August 16, 1973.

AMS (MOS) subject classifications (1970). Primary 28A35; Secondary 46G99, 28A50, 28A60, 28A20, 26A48.

Key words and phrases. Regular Borel probability measure, Baire measures, Baire sets, Borel sets, point and set isometries of measure spaces, Baire and Borel isomorphisms, disintegration of measures, measure algebra, functions monotonic in one variable, evaluation map, measure-preserving transformations.

(1) The author gratefully acknowledges support by the National Science Foundation.

$\left({ }^{2}\right)$ For a somewhat weaker conclusion from more general hypotheses, see [9].

$\left({ }^{3}\right)$ For instance, take $S$ to be the direct sum of th. unit interval $I$ (with Lebesgue measure) and the representation space $R$ of $I$ (with induced measure). The automorphism of the measure algebra of $S$ that interchanges $R$ and $I$ cannot be realized by a pointmapping. For an example in $I^{n}$ see [7]. 
we try to alter $(S, \mu)$, in not too drastic a way, into a space that does have the desirable properties. The method adopted here is suggested by an analogous situation in topology, where it is well known that multiplying a space by a product $\mathbf{R}^{\boldsymbol{w}}$ of copies of the real line tends to "smooth out" the space; under mild assumptions the product becomes homeomorphic to $\mathbf{R}^{\boldsymbol{w}}$ if $\boldsymbol{w}$ is large enough. Analogously we prove here that, under suitable restrictions on $(S, \mu)$, the measure-theoretic product $(S, \mu) \times\left(I^{w}, m^{w}\right)$ is isometric to $\left(I^{w}, m^{w}\right)$ if $w$ is large enough; in fact it is enough to take $w$ to be the weight (that is, the least cardinal of a base of open sets) of the space $S$.

This theorem is deduced from one that applies to a somewhat different class of measure spaces and gives a slightly less precise result-that $(S, \mu) \times\left(I^{w}, m^{w}\right)$ is "pseudo-isometric", in a sense defined below, to $\left(I^{w}, m^{w}\right)$. It would be interesting to know whether the pseudo-isometry here can be replaced by a genuine isometry; I suspect that in general this is not possible.

Another way of looking at these results should perhaps be pointed out, though it is not used in what follows. We can decompose the measure algebra $(E, \mu)$ of $(S, \mu)$ into countably many homogeneous pieces, as in [3]; the $n$th piece is then set-isometric, apart from a scale factor, to the measure algebra of $\left(I^{w}, m^{w_{n}}\right)$ for some cardinal $w_{n}$. Thus if $w$ is large enough it is clear that $(S, \mu) \times\left(I^{w}, m^{w}\right)$ is set-isometric to $\left(I^{w}, m^{w}\right)$; and the above theorems assert, in effect, that here the set-isometry can be realized by a point-mapping.

These theorems, and the techniques used to prove them, can be used to obtain results (which I hope to publish elsewhere) on lifting and disintegration of measures for nonseparable spaces.

Notation. A "pseudo-isometry" $\phi$, from a measure space $\left(S_{1}, B_{1}, \mu_{1}\right)$ to a measure space $\left(S_{2}, B_{2}, \mu_{2}\right)$, is a one-to-one map $\phi$ of $S_{1}$ onto $S_{2}$ such that

(1) for each $E_{2} \in B_{2}, \phi^{-1}\left(E_{2}\right) \in B_{1}$ and $\mu_{1} \phi^{-1}\left(E_{2}\right)=\mu_{2}\left(E_{2}\right)$;

(2) for each $E_{1} \in B_{1}$, there exists $E_{1}^{\prime} \in B_{1}$, differing from $E_{1}$ by a $\mu_{1}$-null set, such that $\phi\left(E_{1}^{\prime}\right) \in B_{2}$.

(Of course (1) shows that we have $\mu_{2} \phi\left(E_{1}^{\prime}\right)=\mu_{1}\left(E_{1}\right)$ in (2).) If in (2) we can always take $E_{1}^{\prime}=E_{1}$ then $\phi$ is an "isometry" (= point-isometry in the sense of [5]).

This definition can be rephrased as follows. Say that a $\sigma$-field of measurable subsets of $S_{1}$ is "full" if it includes at least one member from each measureclass. Then a pseudo-isometry from $\left(S_{1}, B_{1}, \mu_{1}\right)$ to $\left(S_{2}, B_{2}, \mu_{2}\right)$ becomes an isometry if we replace the $\sigma$-field $B_{1}$ of all $\mu_{1}$-measurable sets by a suitable full sub- $\sigma$-field.

The measure space $\left(I^{\aleph 0}, m^{\aleph 0}\right)$-that is, the measure-theoretic product of countably many unit intervals-will arise very often in the course of the work; 
we abbreviate it to $(H, M)$. We shall often use the fact (cf. [11]) that $(H, M)$ and $(I, m)$ are isometric.

We use [1] , [2] as background references for standard measure-theoretic, topological and Borel-set results.

The general strategy underlying the proof of the main theorem is simple. We regard $S$ as embedded topologically in a product space $I^{A}$, where $A$ is a suitable index set. For $B \subset A$, consider the projection $S_{B}$ of $S$ in $I^{B}$, with a measure induced from $\mu$ in a natural way, and suppose we have a suitable pseudo-isometry of $S_{B} \times H^{B}$ onto $H^{B}$. (Of course, $H^{B}$ is essentially the same as $I^{B}$, but it is technically more convenient here.) We "lift" it to a pseudo-isometry of $S_{C} \times H^{C}$ onto $H^{C}$, where $C$ is $B$ together with one extra element of $A$, and continue transfinitely. (Actually a Zorn's Lemma argument will be used, but it comes to the same thing.) We finally reach $B=A$ and have the desired pseudo-isometry of $S \times H^{A}$ onto $H^{A}$.

However, in carrying out this program, difficulties of detail quickly arise, largely in connection with measurability. We do not assume that $S$ has a countable base. Instead, our method for controlling measurability is based on an idea of H. D. Ursell [10]; we make systematic use of functions (from $S_{B} \times I$ to $I)$ that are monotone in the second variable (and measurable in the first). Thus we begin (\$2) with a treatment of increasing functions from $I$ to $I$ and their "pseudo-inverses"; the subject is elementary but $I$ have not seen it considered from the present point of view before. Next $(\S 3)$ we discuss measurability questions for functions from $S_{B} \times I$ to $I$, as mentioned above. Two further preliminary results are needed. One $(\S 4)$ is a theorem on strict disintegration of measures, which may be of independent interest. (It will also furnish the startingpoint for a more general disintegration theorem, as mentioned earlier.) The other exhibits (§5) an explicit isometry of $S \times H$ onto $H$ when $S$ is topologically embedded in $I$. The existence of such an isometry is well known, but we need it in explicit form in order to handle measurablity questions later on in the argument.

The main step comes next ( $\$ 6)$. In the terminology used above (a different notation is used in $\S 6$ ) we have $S_{C} \subset S_{B} \times I$, with suitably related measures, and we wish to construct a suitable isometry of $S_{C} \times H$ onto $S_{B} \times H$ For each $x \in S_{B}$, the results of the two preceding sections enable us to deal with the "section" of $S_{C}$ over $x$; we have only to let $x$ vary, combine the results-and prove measurability. The desired lifting of the pseudo-isometry from $S_{B} \times H^{B} \rightarrow H^{B}$ to $S_{C} \times H^{C} \rightarrow H^{C}$ is then a straightforward consequence (§7). In $\S 8$ we then use Zorn's Lemma to obtain a pseudo-isometry $\phi$ from $S \times H^{A}$ to $H^{A}$, as required.

In $\S 9$ we note a variant of the theorem just proved, obtained by slight revisions of the argument. The result says essentially that if $S$ is $I^{w}$ and $\mu$ is 
a Baire probability measure on $S$, then $(S, \mu) \times\left(I^{w}, m^{w}\right)$ is actually isometric to $\left(I^{w}, m^{w}\right)$, under a correspondence that takes Baire sets to Baire sets (both ways).

Finally, in $\S 10$, we generalize the main theorem (and also the extension theorem in §7) by considering the situation in which we are given two compact Hausdorff spaces $R, S$, a finite Borel measure $\mu$ on $S$, and a continuous map $\theta$ of $S$ onto $R$. Then $\theta$ induces a measure $\lambda$ on $R$. Let $\pi$ be the projection map of $\left(I^{A}, m^{A}\right)$ onto $\left(I^{B}, m^{B}\right)$, where $B \subset A$. We show that, for suitable $A$ and $B$, the product map $\theta \times \pi$ can be converted by pseudo-isometries into the projection map $\pi$.

The author is indebted to A. H. Stone for some helpful discussions, and for assistance with some topological details.

2. Monotone functions and their pseudo-inverses. We are concerned here with monotone maps from one real interval to another; for simplicity we standardize things so that our functions are increasing (= nondecreasing), defined on the unit interval $I$, and with values in $I$. Given such a function $f$, we define its "left and right limit functions", $f_{-}$and $f_{+}$, in a slightly unorthodox way, as follows:

$$
\begin{aligned}
& f_{-}(0)=0 ; \text { if } 0<t \leqslant 1 \text {, then } f_{-}(t)=f(t-)=\lim _{\delta \rightarrow 0+} f(t-\delta) \text {; } \\
& f_{+}(1)=1 ; \text { if } 0 \leqslant t<1 \text {, then } f_{+}(t)=f(t+)=\lim _{\delta \rightarrow 0+} f(t+\delta) .
\end{aligned}
$$

The departure from orthodoxy at 0 and 1 does not affect the familiar facts: $f_{-}, f_{+}$are also increasing functions from $I$ to $I ; f_{-} \leqslant f \leqslant f_{+}$, and $f_{-}(t)=f(t)=f_{+}(t)$ for all but (at most) countably many values of $t \in I$, the "jumps" of $f$ (but it is now easier for 0 and 1 to be jumps); $f_{-}$is continuous on the left, and $f_{+}$is continuous on the right. Also we have the elementary but useful property:

$$
\text { If } 0 \leqslant t<t^{\prime} \leqslant 1 \text { then } f_{+}(t) \leqslant f_{-}\left(t^{\prime}\right) \text {. }
$$

If $f_{-} \leqslant f^{\prime} \leqslant f_{+}$, where $f^{\prime}$ is another map from $I$ to $I$, then $f^{\prime}$ is also increasing, and we have $f_{-}^{\prime}=f_{-}$and $f_{+}^{\prime}=f_{+}$, so that $f_{-}^{\prime} \leqslant f \leqslant f_{+}^{\prime}$. Thus the relation between $f$ and $f^{\prime}$ here is symmetric, and is easily seen to be an equivalence relation (on the set of increasing functions from $I$ to $I$ ). Given $f, f_{-}$is the smallest member of the equivalence class of $f$, and $f_{+}$is the largest; the equivalence class of course consists of all $f^{\prime}: I \rightarrow I$ between them. Thus, on denoting this equivalence relation by $\sim$, we have: if $f \sim f^{\prime}$ then $f_{-}=f_{-}^{\prime}$ and $f_{+}=f_{+}^{\prime}$. In particular, $\left(f_{-}\right)_{-}=f_{-}=\left(f_{+}\right)_{-},\left(f_{-}\right)_{+}=f_{+}=\left(f_{+}\right)_{+}$.

It is easily seen that two increasing maps $f, f^{\prime}$ from $I$ to $I$ are equivalent if and only if $f(t)=f^{\prime}(t)$ for all but countably many values of $t \in I$. However, this way of looking at the equivalence relation will not be particularly 
helpful to us. For our purposes, a more fruitful reformulation is a geometrical one, as follows. Define the "pseudograph" $\Gamma(f)$ of an increasing $f: I \rightarrow I$ to be the subset

$$
\left\{(s, t) \mid f_{-}(s) \leqslant t \leqslant f_{+}(s)\right\} \text { of } I \times I .
$$

(Roughly speaking, this is the ordinary graph of $f$ with the jumps filled in by vertical segments.) Then we have $f \sim f^{\prime}$ if and only if $\Gamma(f)=\Gamma\left(f^{\prime}\right)$.

Given an increasing $f: I \rightarrow I$, if we reflect $\Gamma(f)$ about the diagonal, forming the set $\left\{(s, t) \mid f_{-}(t) \leqslant s \leqslant f_{+}(t)\right\}$, we obtain the pseudograph of another equivalence class of increasing functions from $I$ to $I$; we say these are "pseudoinverse" to $f$ (and to the functions equivalent to $f$ ). Reformulating this in analytic terms, we say that a map $g: I \rightarrow I$ is pseudo-inverse to a given increasing $f: I \rightarrow I$ (or is "a pseudo-inverse of $f$ ") if

$$
f_{+}(g(p)) \geqslant p \geqslant f_{-}(g(p)) \quad(p \in I)
$$

or, equivalently, if (for all $t, p \in I$ )

$$
t>g(p) \Rightarrow f(t) \geqslant p \text { and } t<g(p) \Rightarrow f(t) \leqslant p .
$$

If $g$ is pseudo-inverse to $f$, then (2) shows $f(g(p))=p$ for all but at most countably many values of $g(p)$. Further it is easy to see that $g$ must be increasing, and that (because (3) is really symmetric between $f$ and $g$ ) $f$ is pseudo-inverse to $g$. From (2) the pseudo-inverses of $f$ depend only on the equivalence class of $f$ (and so are determined by $f_{-}$, or by $f_{+}$). Moreover the symmetry of the pseudo-inverse relationship shows that $f, g$ are pseudoinverse if and only if

$$
g_{+}(f(t)) \geqslant t \geqslant g_{-}(f(t)) \quad(t \in I),
$$

which depends only on the equivalence class of $g$.

We define the "upper and lower pseudo-inverses of $f$ " by:

$$
\begin{aligned}
& f^{\leftarrow}(p)=\sup \{t \in I \mid f(t) \leqslant p\}=\inf \{t \in I \mid f(t)>p\}, \\
& f_{\leftarrow}(p)=\inf \{t \in I \mid f(t) \geqslant p\}=\sup \{t \in I \mid f(t)<p\},
\end{aligned}
$$

with the conventions $\sup \varnothing=0, \inf \varnothing=1$. It is easily verified that $f^{\leftarrow}$ and $f_{\leftarrow}$ are indeed pseudo-inverses of $f$, and that

$$
\begin{aligned}
& \text { if } g_{+} \text {is an arbitrary pseudo-inverse of } f \text {, } \\
& \text { then } g_{-}=f_{\leftarrow} \text { and } g_{+}=f^{\leftarrow} \text {. }
\end{aligned}
$$

This confirms that the pseudo-inverses of $f$ form a single equivalence class, having $f^{\leftarrow}$ as its greatest member and $f_{\leftarrow}$ as its smallest member; the pseudoinverses of $f$ constitute precisely all the functions between $f^{\leftarrow}$ and $f_{\leftarrow}$. 
Again, the symmetry of the pseudo-inverse relation shows that $f$ is a pseudo-inverse of $f^{\leftarrow}$ (and of $f_{\leftarrow}$ ). Thus (6) gives $f_{-}=\left(f^{\leftarrow}\right)_{\leftarrow}=\left(f_{\leftarrow}\right)_{\leftarrow}$, $f_{+}=\left(f^{\leftarrow}\right)^{\leftarrow}=\left(f_{\leftarrow}\right)^{\leftarrow}$. Note that from (1) and (6) we have:

$$
\text { If } 0 \leqslant p<p^{\prime} \leqslant 1 \text { then } f^{\leftarrow}(p) \leqslant f_{\leftarrow}\left(p^{\prime}\right) \text {. }
$$

Now, given an increasing $f: I \rightarrow I$, we shall decompose the domain of $f$ into 3 parts, corresponding roughly to: (a) the part for which the pseudograph $\Gamma(f)$ is vertical (that is, $f$ jumps), (b) the part for which $\Gamma(f)$ is horizontal (the intervals on which $f$ is constant), (c) the remaining "good" part, for which both $f$ and its inverse are well behaved. Formally, we define the "jump set" $J(f)$ of $f$ by:

$$
J(f)=\left\{t \in I \mid f_{-}(t)<f_{+}(t)\right\},
$$

and the "set of values of constancy" $K(f)$ of $f$ by:

$$
K(f)=\left\{p \in I \mid f^{-1}(p) \text { has more than one point }\right\} .
$$

For each $p \in K(f), f^{-1}(p)$ is an interval of positive length, which may or may not include one or both of its endpoints; we denote the closure of this interval (including both endpoints) by $\mathrm{Cl}\left(f^{-1}(p)\right)$. We note for later use that if $f$ is continuous on the right then the interval $f^{-1}(p)(p \in K(f))$ certainly contains its left-hand endpoint, and similarly for the right-hand endpoint if $f$ is continuous on the left.

Clearly if $f \sim f^{\prime}$ we have

$$
J(f)=J\left(f^{\prime}\right) \text { and } K(f)=K\left(f^{\prime}\right) .
$$

Now define the "constancy set" $C(f)$ of $f$ by:

$$
C(f)=\bigcup\left\{f^{-1}(p) \mid p \in K(f)\right\}=f^{-1}(K(f)),
$$

and the "extended constancy set" $C^{\prime}(f)$ of $f$ by:

$$
C^{\prime}(f)=\bigcup\left\{\mathrm{Cl}\left(f^{-1}(p)\right) \mid p \in K(f)\right\} .
$$

It is easy to see that

$$
C(f) \subset C^{\prime}(f) \subset C(f) \cup J(f),
$$

and that the restriction $f \mid I-C(f)$ is strictly increasing (and hence one-to-one).

If $f \sim f^{\prime}$ then for each $p \in K(f)$ we see that the intervals $f^{-1}(p)$ and $\left(f^{\prime}\right)^{-1}(p)$ have the same interiors, so

$$
C^{\prime}(f)=C^{\prime}\left(f^{\prime}\right) \text {. }
$$

Further, because $K(f)=K\left(f_{-}\right)=K\left(f_{+}\right)$and $f_{-}, f_{+}$are continuous on the 
left and right respectively, we see that if $p \in K(f)$ then $\left(f_{-}\right)^{-1}(p) \cup\left(f_{+}\right)^{-1}(p)$ contains both endpoints of the interval $f^{-1}(p)$, so that

$$
C^{\prime}(f)=C\left(f_{-}\right) \cup C\left(f_{+}\right) .
$$

Finally we define $G(f)$, the "good set" for $f$, by

$$
G(f)=I-\left(J(f) \cup C^{\prime}(f)\right)=I-(J(f) \cup C(f))
$$

(from (9)); from the foregoing, this too depends only on the equivalence class of $f$.

Now suppose that $g$ is any pseudo-inverse of the increasing function $f: I \rightarrow I$, for instance $g=f^{\leftarrow}$ or $f_{\leftarrow}$. It is easy to verify that

$$
\begin{aligned}
& K(g)=J(f) \text {, and for each } t \in K(g) \text { the interior } \\
& \text { of the interval } g^{-1}(t) \text { is }\left(f_{-}(t), f_{+}(t)\right) \text {. }
\end{aligned}
$$

But since $f$ is also a pseudo-inverse of $g$, (12) gives:

$$
K(f)=J(g) \text {, and for each } p \in K(f) \text { the interior }
$$

of the interval $f^{-1}(p)$ is $\left(g_{-}(p), g_{+}(p)\right)=\left(f_{\leftarrow}(p), f^{\leftarrow}(p)\right)$.

It follows that $K(f)$ and $K(g)$ are countable sets (as is also clear directly).

A straightforward verification now shows that, if $f$ and $g$ are mutually pseudo-inverse increasing functions from $I$ to $I$, the restriction of $f$ to $G(f)$ is a continuous, strictly increasing map of $G(f)$ onto $G(g)$; the inverse map is the restriction $g \mid G(g)$ (which is also, of course, a continuous and strictly increasing map of $G(g)$ onto $G(f)$ ). In particular, $f \mid G(f)$ and $g \mid G(g)$ are genuinely inverse.

We shall later need the identity (for increasing $f: I \rightarrow I$ ):

$$
f(I)=\left(I-\cup\left\{\left[f_{-}(t), f_{+}(t)\right] \mid t \in J(f)\right\}\right) \cup f(J(f)) \cup K(f) .
$$

To prove this, take an arbitrary pseudo-inverse $g$ of $f$; then

$$
\begin{aligned}
f(I) & =f(G(f)) \cup f(J(f)) \cup f(C(f)) \\
& =G(g) \cup f(J(f)) \cup K(f) \\
& =\left(I-\left(J(g) \cup C^{\prime}(g)\right)\right) \cup f(J(f)) \cup K(f),
\end{aligned}
$$

and the result follows from (12) and (13).

Finally, we note that all the sets considered here $-J(f), K(f), C(f)$, $C^{\prime}(f)$ and $G(f)$-are Borel sets in $I$. For $J(f)$ and $K(f)\left(=J\left(f^{\leftarrow}\right)\right)$ are countable; hence $C(f)$ and $C^{\prime}(f)$ are $F_{\sigma}$ sets, and $G(f)$ is $G_{\delta}$.

3. Some lemmas on measurability.

3.1. Suppose that $X, Y, Z$ are arbitrary sets, and that $B(X), B(Y)$, $B(Z)$ are given $\sigma$-fields of subsets of $X, Y, Z$ respectively. We define $B(X \times Y)$ 
to be the product $\sigma$-field $B(X) \times B(Y)$-that is, the $\sigma$-field of subsets of $X \times Y$ generated by the sets $A \times B(A \in B(X), B \in B(Y))$-and define $B(X \times Z)$ similarly. A function $f: X \rightarrow Y$ is said to be $B$-measurable if $f^{-1}(B) \in B(X)$ for all $B \in B(Y)$; and similarly we define the $B$-measurability of $F: X \times Y \rightarrow Z$ and of $\widetilde{F}: X \times Y \rightarrow X \times Z$ (requiring, for instance, that $\widetilde{F}^{-1}(D) \in B(X \times Y)$ for all $D \in B(X \times Z))$. Then we have:

If $F: X \times Y \rightarrow Z$ is B-measurable, and $\widetilde{F}: X \times Y \rightarrow X \times Z$ is defined by $\widetilde{F}(x, y)=(x, F(x, y))$, then $\widetilde{F}$ is B-measurable.

For it suffices to show that $\widetilde{F}^{-1}(A \times C) \in B(X \times Y)$ for all $A \in B(X)$ and $C \in B(Z)$; but $\widetilde{F}^{-1}(A \times C)=(A \times Y) \cap F^{-1}(C) \in B(X \times Y)$.

3.2. Now we specialize by taking $Y=Z=I$, the unit interval, with $B(I)$ $=$ the usual $\sigma$-field of Borel subsets of $I$. Suppose $F: X \times I \rightarrow I$ satisfies the following conditions:

(1) For each $t \in I$, the map $F^{t}: X \rightarrow I$, where $F^{t}(x)=F(x, t)$, is B-measurable.

(2) For each $x \in X$, the map $F_{x}: I \rightarrow I$, where $F_{x}(t)=F(x, t)$, is increasing (that is, nondecreasing).

(3) Either (a) $F_{x}$ is continuous on the left (that is, $F_{x}(t-)=F_{x}(t)$ for $0<t \leqslant 1$ ) for all $x \in X$, or

(b) $F_{x}$ is continuous on the right for all $x \in X$.

Then we show $\left({ }^{4}\right)$ :

THEOREM. $F$ is B-measurable; and consequently (by the remark in 3.1) so is $\widetilde{F}: X \times I \rightarrow X \times I$, where $\widetilde{F}(x, t)=(x, F(x, t))$.

First suppose condition (3a) is satisfied (as well as conditions (1) and (2)). Fixing an arbitrary $p \in I$, it will be enough to show that the set $E=$ $\{(x, t) \mid x \in X, t \in I, F(x, t) \leqslant p\}$ is in $B(X \times I)$. For each $x \in X$, define

$$
\xi(x)=\sup \{t \in I \mid F(x, t) \leqslant p\},
$$

with the conventions sup $\varnothing=0$, inf $\varnothing=1$ as before; note that $\xi(x)=$ $\left(F_{x}\right)^{\leftarrow}(p)$, in the notation of $\S 2$. The first step is to show that $\xi: X \rightarrow I$ is $B$-measurable. For each rational $\rho \in I$, put $X_{\rho}=\{x \in X \mid F(x, \rho) \leqslant p\}$; from (1), $X_{\rho} \in B(X)$. Put $g_{\rho}=\rho$ times the characteristic function of $X_{\rho}$, and define $g: X \rightarrow I$ by

$$
g(x)=\sup \left\{g_{\rho}(x) \mid \rho \text { rational, } 0 \leqslant \rho \leqslant 1\right\} \quad(x \in X) .
$$

Clearly $g_{\rho}$ is B-measurable; hence so is $g$. But it is not hard to see from (2) that $g=\xi$, which is therefore $B$-measurable.

(4) This theorem generalizes results of Ursell [10]. 
Now define $E_{0}=\{x \in X \mid F(x, 0) \leqslant p\}$ and, for $n=1,2, \cdots$ and $k=1,2, \cdots, n$,

$$
\begin{aligned}
& H_{k n}=\{x \in X \mid(k-1) / n \leqslant \xi(x) \leqslant k / n\}, \\
& W_{k n}=H_{k n} \times[0, k / n] .
\end{aligned}
$$

We verify that

$$
E=\left(E_{0} \times I\right) \cap \bigcap_{n=1}^{\infty} \bigcup_{k=1}^{n} w_{k n}
$$

In fact, if $(x, t) \in E$ then $t \leqslant \xi(x)$; also, by (2), we have $F(x, 0) \leqslant$ $F(x, t) \leqslant p$, so $(x, t) \in E_{0} \times I$. For each $n=1,2, \cdots$, there is at least one integer $k$ such that $1 \leqslant k \leqslant n$ and $(k-1) / n \leqslant \xi(x) \leqslant k / n$. Thus $x \in H_{k n}$ and $t \leqslant k / n$, so $(x, t) \in W_{k n}$, proving that $(x, t)$ is in the set on the right of (*). Conversely, if $(x, t)$ is in that set, we take for each $n=1,2, \cdots$ a corresponding $k$ for which $(x, t) \in W_{k n}$; that is, $(k-1) / n \leqslant \xi(x) \leqslant k / n$ and $t \leqslant k / n$. This shows that $t \leqslant \xi(x)+1 / n$ for all $n$, proving that $t \leqslant \xi(x)$. If $t=0$, we have $F(x, t)=F(x, 0) \leqslant p$ (because $\left.x \in E_{0}\right)$, showing $(x, t)$ $\in E$. So we may assume $0<t \leqslant \xi(x)(\leqslant 1)$. From (2) we have $F(x, t) \leqslant$ $F(x, \xi(x))$, which in turn $\leqslant p$ because of condition (3a). Thus again $(x, t) \in E$.

From (*) it is clear that $E \in B(X \times I)$, as required.

3.3. Now suppose $F$ satisfies conditions (1), (2) and (3b). Fixing a positive integer $n$ for the present, we define

$$
\begin{aligned}
& \tau_{n}(t)=\min (t+1 / n, 1) \quad(t \in I), \\
& H_{n}(x, t)=F_{x}\left(\tau_{n}(t)-\right)=\lim _{\delta \rightarrow 0+} F\left(x, \tau_{n}(t)-\delta\right) .
\end{aligned}
$$

From the fact that $F$ satisfies conditions (1) and (2), it follows that $H_{n}$ satisfies (1), (2) and (3a); we omit the verification of this, which is straightforward. Hence the foregoing shows that $H_{n}$ is $B$-measurable. So, therefore, is $\inf _{n} H_{n}$; but another routine verification shows that (because $F$ satisfies condition (3b)) inf $_{n} H_{n}(x, t)=F(x, t)$ if $0 \leqslant t<1$, and from this and (1) the measurability of $F$ follows easily.

3.4. We remark (cf. $[10$, p. 330]) that condition (1) above can be weakened; it suffices that it hold for a dense set of values of $t$ including 0 in case (a), 1 in case (b). This is shown by the proof, where the rational numbers could equally well be replaced by a countable dense subset of the dense set in question; but it also follows from the observation that the original form of condition (1) is an easy consequence of the weakened form of it, in the presence of conditions (2) and (3). 
However, the continuity condition (3) is essential.(5) For example, suppose $X=I$, and in $I \times I$ let $P=\{(x, t) \mid x+t>1\}$, and let $Q$ be an arbitrary non-Borel subset of the segment $\{(x, t) \mid x+t=1\}$. Then the characteristic function $F$ of $P \cup Q$ satisfies (1) and (2), and moreover $F_{x}$ is continuous on one side or the other for each $x$; but $F$ is not $B$-measurable.

3.5. Now suppose $F: X \times I \rightarrow I$ satisfies condition (2) above: each $F_{x}: I \rightarrow I$ is increasing $(x \in X)$. We apply the considerations of $\S 2$ to $f=F_{x}$, adopting the following notation. $F_{-}(x, t)$ denotes $\left(F_{x}\right)_{-}(t)$; that is, $F_{-}(x, 0)=0$ and $F_{-}(x, t)=\lim _{\delta \rightarrow 0+} F(x, t-\delta)$ for $0<t \leqslant 1$. Similarly $F_{+}(x, t)$ denotes $\left(F_{x}\right)_{+}(t)$, and we write $F_{\leftarrow}(x, t)$ for $\left(F_{x}\right)_{\leftarrow}(t)$ and $F^{\leftarrow}(x, t)$ for $\left(F_{x}\right)^{\leftarrow}(t)$. In other words, $x$ plays a purely passive role throughout. Analogously we define the "jump set" of $F$ by

$$
J^{*}(F)=\left\{(x, t) \mid t \in J\left(F_{x}\right)\right\}=\left\{(x, t) \mid F_{+}(x, t)>F_{-}(x, t)\right\}
$$

(where it is understood that $x \in X$ and $t \in I$ ), and (where $p \in I$ )

$$
\begin{aligned}
& K^{*}(F)=\left\{(x, p) \mid p \in K\left(F_{x}\right)\right\}=\left\{(x, p) \mid\left(F_{x}\right)^{-1}(p) \text { has } \geqslant 2 \text { points }\right\} \\
& C^{*}(F)=\left\{(x, t) \mid t \in C\left(F_{x}\right)\right\}=\widetilde{F}^{-1}\left(K^{*}(F)\right),
\end{aligned}
$$

the "constancy set" of $F$, where $\widetilde{F}$ is defined as in 3.1,

$$
C^{* *}(F)=\left\{(x, t) \mid t \in C^{\prime}\left(F_{x}\right)\right\}
$$

the "extended constancy set" of $F$, and

$$
G^{*}(F)=\left\{(x, t) \mid t \in G\left(F_{x}\right)\right\}=(X \times I)-\left(J^{*}(F) \cup C^{* *}(F)\right),
$$

the "good set" for $F$.

The results of $\$ 2$ apply immediately to these sets and functions; one merely has to think of $x$ as being fixed. Thus, for instance, $\left(F^{\leftarrow}\right)^{\leftarrow}=F_{+}, J^{*}(F)=$ $K^{*}\left(F^{\leftarrow}\right)=J^{*}\left(F_{\leftarrow}\right)$, and so on.

The principal goal of this section is the following result.

Lemma. Suppose $F: X \times I \rightarrow I$ satisfies conditions (1) and (2) of 3.2 above; then all the functions $F_{-}, F_{+}, F^{\leftarrow}, F_{\leftarrow}$ and sets $J^{*}(F), K^{*}(F), C^{\prime *}(F)$ and $G^{*}(F)$ are B-measurable.

(We remark that condition (1) could be weakened, as noted above. Note that condition (3) is not assumed, and that nothing is said about $C^{*}(F)$.) $\left({ }^{6}\right)$.

(5) For B-measurability of $F$. As shown in [10], (3) is not needed to ensure Lebesgue measurability, for instance when $X=I$.

(6) An adaptation of the example in 3.4 shows that $C^{*}(F)$ need not be in $B(X \times I)$ in general. 
Proof. Clearly $F_{-}$also satisfies conditions (1) and (2), and in addition it satisfies (3a). So, by the theorem in 3.2, $F_{-}$is B-measurable. Similarly, so is $F_{+}$(which satisfies (3b)). To see that $F^{\leftarrow}$ is $B$-measurable, we show that it satisfies conditions (1), (2) and (3b). Condition (1) has been verified in 3.2, in showing that $\xi$ was $B$-measurable; for $\xi(x)=\left(F_{x}\right)^{\leftarrow}(p)=F^{\leftarrow}(x, p)$ (with $p$ fixed but arbitrary). Condition (2) is clear, because for each fixed $x \in X$, $F^{\leftarrow}(x, p)$ is an increasing function of $p$. And from equation $2(6)$ we see that $F^{\leftarrow}(x, p)=\left(F_{\leftarrow}(x, p)\right)_{+}$and is thus (for each fixed $\left.x \in X\right)$ continuous on the right, so (3b) holds. Similarly $F_{\leftarrow}$ is $B$-measurable.

Since $F_{+}$and $F_{-}$are $B$-measurable, it is clear from the definition of $J^{*}$ that $J^{*}(F) \in B(X \times I)$. Again, $\S 2$ shows that $K^{*}(F)=J^{*}\left(F^{*}\right)$, and thus $K^{*}(F) \in B(X \times I)$.

It follows that if $F$ happens to be $B$-measurable, then $\widetilde{F}^{-1}\left(K^{*}(F)\right) \in$ $B(X \times I)$-that is, $C^{*}(F)$ is then in $B(X \times I)$. But, whether or not $F$ is $B$-measurable, we have seen that $F_{-}$and $F_{+}$are. Hence $C^{*}\left(F_{-}\right)$and $C^{*}\left(F_{+}\right)$are in $B(X \times I)$. Their union is just $C^{* *}(F)$, from equation 2(11), which proves $C^{\prime *}(F) \in B(X \times I)$. Finally,

$$
G^{*}(F)=(X \times I)-\left(J^{*}(F) \cup C^{* *}(F)\right) \in B(X \times I) .
$$

REMARK. From $\S 2$, the restriction $F \mid G^{*}(F)$ coincides with both $F_{-} \mid G^{*}(F)$ and $F_{+} \mid G^{*}(F)$, and so is $B$-measurable; and it is a bijection of $G^{*}(F)$ onto $G^{*}\left(F^{\leftarrow}\right)=G^{*}\left(F_{\leftarrow}\right)$; the inverse map is $F^{\leftarrow}\left|G^{*}\left(F^{\leftarrow}\right)=F_{\leftarrow}\right| G^{*}\left(F^{\leftarrow}\right)$, which is likewise $B$-measurable.

3.6. We need one further elementary result:

Suppose $f: X \rightarrow I$ is B-measurable; then the sets $\{(x, t) \mid x \in X, t \in I$, $t<f(x)\}$ and $\{(x, t) \mid x \in X, t \in I, t \leqslant f(x)\}$ are both in $B(X \times I)$.

This is easy to prove directly, but it can be deduced from 3.2 as follows.

Let $\mathrm{R}$ denote the real line, and define $G: X \times \mathbf{R} \rightarrow \mathbf{R}$ by $G(x, t)=f(x)-t$. The theorem of 3.2 (which applies if $\mathrm{R}$ replaces $I$, with substantially the same proof) shows that $G$ is $B$-measurable; then $G^{-1}(0, \infty) \in B(X \times \mathbf{R})$, and the intersection of this set with $X \times I$ is therefore in $B(X \times I)$. But this intersection is $\{(x, t) \mid x \in X, t \in I, t<f(x)\}$. The proof for the second set is similar.

\section{A disintegration theorem.}

4.1. Throughout this section, $R$ is a compact Hausdorff space, $S$ is a closed (hence compact) subset of $R \times I$ such that the projection map $\pi: R \times I \rightarrow R$ maps $S$ onto all of $R$, and $\mu$ is a regular Borel measure on $S$, with $\mu(S)=1$, completed with respect to null sets. We use the following notation. $B(R), B(R \times I), B(S), B(I)$ denote the respective families of Borel 
subsets of $R, R \times I, S, I$. (This is consistent with the notation of 3.1; $B(R \times I)$ coincides with the product $\sigma$-field $B(R) \times B(I)$, because $I$ has a countable base of open sets.) For each $r \in R$ and $P \subset R \times I$, we put $P_{r}=\{t \in I \mid(r, t) \in P\}$, so that $\{r\} \times P_{r}$ is the "section" $P \cap \pi^{-1}(r)$, which we denote by $P^{r}$. The restriction $\pi \mid S$ is denoted by $\theta$; thus $S^{r}=\{r\} \times S_{r}=$ $\theta^{-1}(r)$.

We define a Borel measure $\lambda$ on $R$ (easily seen to be regular and complete with respect to null sets) by setting $\lambda=\mu \cdot \theta^{-1}$; that is, $\lambda(E)=\mu\left(\theta^{-1}(E)\right)=$ $\mu(S \cap(E \times I))$ for Borel (or merely measurable) $E \subset R$.

THEOREM. Under the above hypotheses, we can define a Borel measure $\mu_{r}$ on each $S_{r}(r \in R)$, in such a way that: (i) $\mu_{r}\left(S_{r}\right)=1$, (ii) for all $P \in B(S)$, the map $r \mapsto \mu_{r}\left(P_{r}\right)$ (of $R$ in $I$ ) is Borel measurable, (iii) for each Borel (or measurable) subset $A$ of $R, \mu(P \cap(A \times I))=\int_{A} \mu_{r}\left(P_{r}\right) d \lambda(r)$. In particualr, $\mu(P)=\int_{R} \mu_{r}\left(P_{r}\right) d \lambda(r)$.

Note that this theorem gives a "strict disintegration" in the sense that each measure $\mu_{r}$ is defined (in effect) on the corresponding section $S^{r}$. It is related to results in [8] (in particular, [8, Theorem 2, p. 136]), but does not seem to follow easily from them. The measures $\mu_{r}$ are automatically regular, because $S_{r} \subset I$.

4.2. Proof. The proof consists in adapting familiar measure-theoretic techniques to a less familiar situation. For convenience we extend $\mu$ to a regular Borel measure (also denoted by $\mu$ ) on all of $R \times I$, by defining $\mu$ to be 0 outside $S$. For each $H \in B(R \times I)$, consider the set function $\mu_{H}$ on $B(R)$ defined by

$$
\mu_{H}(A)=\mu(H \cap(A \times I)) \quad(A \in B(R)) ;
$$

this is a countably additive measure on $R$, absolutely continuous with respect to $\lambda$ (and in fact majorized by $\lambda$ ). So it has a Radon-Nikodym derivative $f_{H}$; in other words, $f_{H}$ is a Borel measurable function on $R$ such that, for each $A \in B(R)$,

$$
\mu(H \cap(A \times I))=\int_{A} f_{H}(r) d \lambda(r) .
$$

This determines $f_{H}$ only modulo $\lambda$-null sets; we choose one such $f_{H}$ arbitrarily for each $H$. Clearly $0 \leqslant f_{H}(r) \leqslant 1$ a.e. To facilitate printing, we also write $f_{H}(r)$ as $f(H, r)$.

For each $H \in B(R \times I)$, define its "essential projection" by:

$$
p(H)=\{r \in R \mid f(H, r)>0\} .
$$


We observe that, although $\pi(S \cap H)$ need not be measurable,

$$
\lambda(p(H)-\pi(S \cap H))=0 \quad(H \in B(R \times I)) .
$$

For the regularity of $\mu$ shows that there exists an $F_{\sigma}$ set $F \subset S \cap H$ with $\mu(F)=\mu(S \cap H)=\mu(H)$, and it suffices to show that $\lambda(p(H)-\pi(F))=$ 0 . But $\pi(F)$ is $\sigma$-compact, hence Borel; thus if $p(H)-\pi(F)$ is not $\lambda$-null it contains a Borel set $A$ of positive $\lambda$-measure on which $f(H, r)$ is bounded away from 0 . Then

$$
\mu(F \cap(A \times I))=\mu(H \cap(A \times I))=\int_{A} f_{H}(r) d \lambda(r)>0,
$$

contradicting the fact that $F \cap(A \times I)=\varnothing$.

Our main object is to arrange, by discarding a suitable null set in $R$, that $f(H, r)$ behaves for fixed $r$ like a measure on a judiciously chosen family of sets $H$. Let $F$ denote the finitely additive field of subsets of $I$ generated by the (not necessarily open or closed) intervals with rational endpoints, and put $G=\{(R \times F) \mid F \in F\}$; this is a finitely additive field of Borel subsets of $R \times I$. Since $G$ is countable, there is a $\lambda$-null Bọrel set $N \subset R$ such that, for all $r \in$ $R-N$, we have:

$$
\begin{aligned}
& f(\varnothing, r)=0, f(S, r)=1=f(R \times I, r), \text { and } \\
& 0 \leqslant f(G, r) \leqslant 1 \text { for all } G \in G ;
\end{aligned}
$$

if $G_{1}, G_{2}, \cdots, G_{k}$ are pairwise disjoint members of $G$, then $f\left(G_{1} \cup \cdots \cup G_{k}, r\right)=f\left(G_{1}, r\right)+\cdots+f\left(G_{k}, r\right)$;

for all $G \in G, p(G) \subset \pi(S \cup G) \cup N$.

We next wish to extend (4) to certain countable (disjoint) unions. To do, this, let $F^{\prime} \subset F$ be the family of all sets $F^{\prime}$ expressible as the union of a finite number of disjoint open rational intervals in $I$ (open relative to $I$ ), and let $G^{\prime} \subset G$ be the family of all sets $G^{\prime}=R \times F^{\prime}$ where $F^{\prime} \in F^{\prime}$. We show that, for each $G \in G$ and almost all $r \in R-N$,

$$
f(G, r)=\inf \left\{f\left(G^{\prime}, r\right) \mid G^{\prime} \in G^{\prime} \text { and } G^{\prime} \supset G\right\} .
$$

For (4) gives $\leqslant$ here. To prove $\geqslant$, note that each $F \in F$ is a finite union of disjoint rational-ended intervals (not necessarily open or closed), and hence $F$ is expressible as $\bigcap\left\{F_{n}^{\prime} \mid n=1,2, \cdots\right\}$ where $F_{n}^{\prime} \in F^{\prime}$ and $F_{1}^{\prime} \supset F_{2}^{\prime} \supset \cdots$. Thus, given $G \in G$, we have $G=\bigcap_{n} G_{n}^{\prime}$ where $G_{n}^{\prime} \in G^{\prime}$ and $G_{1}^{\prime} \supset G_{2}^{\prime} \supset \cdots$. From (4) again, $f\left(G_{1}^{\prime}, r\right) \geqslant f\left(G_{2}^{\prime}, r\right) \geqslant \cdots$; let $\lim _{n \rightarrow \infty} f\left(G_{n}^{\prime}, r\right)=g(r)$. It will suffice to prove $g(r) \leqslant f(G, r)$ a.e. But if not, there exist $\epsilon>0$ and a Borel subset $A$ of $R-N$ with $\lambda(A)>0$ such that, for each $r \in A, g(r)>f(G, r)+\epsilon$. A routine calculation, using (1), shows 
$\mu\left(\left(G_{n}^{\prime}-G\right) \cap(A \times I)\right) \geqslant \epsilon \lambda(A)$ for all $n$. But $\mu\left(G_{n}^{\prime}-G\right) \rightarrow 0$ as $n \rightarrow \infty$, giving a contradiction.

Since $G$ is countable, we can enlarge the null set $N$ to $N^{\prime}$ so as to make (6) hold for all $G \in G$ and all $r \in R-N^{\prime}$.

Similarly, on writing $G^{\prime \prime}$ for the family of all sets $R \times F^{\prime \prime}$ where $F^{\prime \prime}$ is the union of a finite number of disjoint closed rational intervals (possibly degenerate) in $I$, and noting that each $F \in F$ is a countable increasing union of sets like $F^{\prime \prime}$, we obtain:

$$
f(G, r)=\sup \left\{f\left(G^{\prime \prime}, r\right) \mid G^{\prime \prime} \in G^{\prime \prime} \text { and } G^{\prime \prime} \subset G\right\} \text {, }
$$

$$
\text { for all } G \in G \text { and all } r \in R-N^{\prime \prime} \text {, where } N^{\prime \prime}\left(\supset N^{\prime} \supset N\right)
$$

is a $\lambda$-null Borel set.

Now we obtain the desired extension of (4):

$$
\text { If } G=\bigcup_{i=1}^{\infty} G_{i} \text {, where } G_{1}, G_{2}, \cdots \text {, and } G \text { are in } G
$$
and $G_{1}, G_{2}, \cdots$ are pairwise disjoint, and if $r \in R-N^{\prime \prime}$, then $f(G, r)=\sum_{i=1}^{\infty} f\left(G_{i}, r\right)$.

For, given $\epsilon>0$, we use (6) and (7) to find $G_{i}^{\prime} \in G^{\prime}$ and $G^{\prime \prime} \in G^{\prime \prime}$ such that $G_{i}^{\prime} \supset G_{i}, G^{\prime \prime} \subset G, f\left(G^{\prime \prime}, r\right)>f(G, r)-\epsilon / 2$, and $f\left(G_{i}^{\prime}, r\right)<f\left(G_{i}, r\right)+$ $\epsilon / 2^{i+1}\left(i=1,2, \cdots ; r\right.$ is fixed for the moment). Since $G^{\prime \prime}$ is compact and each $G_{i}^{\prime}$ is open, we have $G^{\prime \prime} \subset G_{1}^{\prime} \cup \cdots \cup G_{n}^{\prime}$ for some $n$. It follows easily from (4) that $f\left(G^{\prime \prime}, r\right) \leqslant f\left(G_{1}^{\prime}, r\right)+\cdots+f\left(G_{n}^{\prime}, r\right)$; thus $f(G, r) \leqslant \Sigma_{i} f\left(G_{i}, r\right)+\epsilon$. Since $\epsilon$ is arbitrary, this proves one inequality in (8); and the other is a trivial consequence of (4).

Now for $r \in R-N^{\prime \prime}$ and $F \in F$, put $r F=F \cap S_{r}$; the family $r F=$ $\left\{{ }^{r} F \mid F \in F\right\}$ is thus a finitely additive field of subsets of $S_{r}$. We have:

$$
\begin{aligned}
& \text { If } r \in R-N^{\prime \prime}, F_{1}, F_{2} \in F \text { and }{ }^{r} F_{1}={ }^{r} F_{2} \text {, then } \\
& f\left(R \times F_{1}, r\right)=f\left(R \times F_{2}, r\right) \text {. }
\end{aligned}
$$

For let $G_{1}=R \times F_{1}, G_{2}=R \times F_{2}$; then $f\left(G_{1}-G_{2}, r\right)=0$ since otherwise $r \in p\left(G_{1}-G_{2}\right)$ and therefore (by (5)) there exists $t \in I$ such that $(r, t) \in\left(G_{1}-G_{2}\right) \cap S$. But then $t \in{ }^{r} F_{1}-{ }^{r} F_{2}$, which is impossible. Hence, by (4), $f\left(G_{1}, r\right)=f\left(G_{1} \cap G_{2}, r\right)$. Similarly $f\left(G_{1} \cap G_{2}, r\right)=f\left(G_{2}, r\right)$, establishing (9).

Because of (9) we can consistently define, for ${ }^{r} F \in{ }^{r} F$ and $r \in R-N^{\prime \prime}$,

$$
\mu_{r}(r F)=f(R \times F, r),
$$

where $F$ is any member of $F$ such that ${ }^{r} F=F \cap S_{r}$. From (3) we have $\mu_{r}(\varnothing)=0, \mu_{r}\left(S_{r}\right)=1,0 \leqslant \mu_{r}\left({ }^{r} F\right) \leqslant 1$. It is not hard to see from (4) and (5) that $\mu_{r}$ is finitely additive. A similar argument, using (8) instead of (4), shows 
that $\mu_{r}$ is countably additive on ${ }^{r} F$. Hence $\mu_{r}$ can be extended to a countably additive measure (which we still denote by $\mu_{r}$ ) on the $\sigma$-field of subsets of $S_{r}$ generated by ${ }^{r} F$. But this is precisely the $\sigma$-field $B\left(S_{r}\right)$ of all Borel subsets of $S_{r}$.

Next we define $\mu_{r}$ when $r \in N^{\prime \prime}$. (Of course this has no measure-theoretic significance, but some care is needed here to ensure good Borel behavior.) Recall that, for each $r \in R, S_{r}$ is a compact nonempty subset of $I$; let $a_{r}$ be its smallest member. For each $r \in N^{\prime \prime}$, take $\mu_{r}$ to be the corresponding pointmeasure concentrated at $a_{r}$ (that is, if $E \in B\left(S_{r}\right)$, then $\mu_{r}(E)=1$ if $a_{r} \in E$, 0 otherwise).

4.3. We still have to show that the properties (ii) and (iii) of the theorem are valid. Let $P$ be the family of all $P^{\prime} \in B(R \times I)$ such that the assertions (ii) and (iii) hold for $P=P^{\prime} \cap S$ (and all $A \in B(R)$ ). It will suffice to show that $P=B(R \times I)$. This requires a number of steps.

(a) If $F \in F$, then $R \times F \in P$.

Put $P=(R \times F) \cap S$; then, for $r \in R-N^{\prime \prime}$ we have $\mu_{r}\left(P_{r}\right)=f(R \times F, r)$, which is a $B$-measurable function of $r$ on $R-N^{\prime \prime}$. But if $r \in N^{\prime \prime}$ we have $\mu_{r}\left(P_{r}\right)=1$ if $\left(r, a_{r}\right) \in P, 0$ otherwise; and since $\left\{r \in R \mid a_{r} \in F\right\}$ is a Borel set (because $a_{r}$ is a lower semicontinuous function of $r$ ) the assertion (ii) follows. And (iii) follows from (1) since $\lambda\left(N^{\prime \prime}\right)=0$.

(b) If $P \in P$ then $(R \times I)-P \in P$.

For if $Q=(R \times I)-P$ then $\mu_{r}\left(Q_{r}\right)=1-\mu_{r}\left(P_{r}\right)$ for all $r \in R$. A similar but slightly more elaborate calculation shows:

(b') If $P, Q \in P$ and $Q \supset P$, then $Q-P \in P$.

(c) If $P_{1}, P_{2}, \cdots \in P$ and $P_{1} \subset P_{2} \subset \cdots$ then $U_{n} P_{n} \in P$.

Here (ii) is immediate, and (iii) follows from the "monotone convergence theorem" for integrals.

(d) If $P_{1}, P_{2}, \cdots \in P$ and $P_{1} \supset P_{2} \supset \cdots$ then $\bigcap_{n} P_{n} \in P$.

From (b) and (c).

(e). If $P_{1}, P_{2}, \cdots$ are disjoint and $P_{n} \in P(n=1,2, \cdots)$ then $\bigcup_{n} P_{n} \in P$.

For if $P=\bigcup_{n} P_{n}$ we have $\mu_{r}(P)=\Sigma_{n} \mu_{r}\left(P_{n}\right)$ for each $r \in R$.

(f) If $B \in B(R)$ and $P \in P$ then $P \cap(B \times D) \in P$.

For if $Q=P \cap(B \times I)$ we have $\mu_{r}(Q)=\mu_{r}(P) \chi_{B}(r)$, where $\chi_{B}$ is the characteristic function of $B$.

(g) If $B \in B(R)$ and $F \in F$ then $B \times F \in F$.

From (a) and (f).

(h). If $U$ is open in $R \times I$, then $U \in P$.

Enumerate the rational open intervals of $I$ (open relative to $I$ ) as $W_{1}$, $W_{2}, \cdots$. For $n=1,2, \cdots$, let $V_{n}$ be the set of all $r \in R$ having an open 
neighborhood $V(r)$ in $R$ such that $V(r) \times W_{n} \subset U$. Then $V_{n}$ is open, and it is easy to see that $U=U_{n}\left(V_{n} \times W_{n}\right)$. From this one sees that $U$ can be written as the union of a countable disjoint family of sets, each of the form $B \times F$ with $B \in B(R), F \in F$. For $V_{1} \times W_{1}$ is already of the form $B \times F$; the difference $\left(V_{2} \times W_{2}\right)-\left(V_{1} \times W_{1}\right)$ is the union of 2 disjoint sets of this form (namely $\left(V_{1} \cap V_{2}\right) \times\left(W_{2}-W_{1}\right)$ and $\left.\left(V_{2}-V_{1}\right) \times W_{2}\right)$, and so on; at the $n$th stage we express $\left(V_{n} \times W_{n}\right)-\left(\left(V_{1} \times W_{1}\right) \cup \cdots \cup\left(V_{n-1} \times W_{n-1}\right)\right)$ as the union of $n$ disjoint sets of this form. The result (h) now follows from (g) and (e).

4.4. The desired conclusion $P=B(R \times I)$ now follows from the following lemma (a variant of the "monotone family theorem" [1, p. 27]), which is often useful in such situations.

Lemma. Let A be a family of subsets of a set $X$, containing $X$ and closed under finite intersections. Let $P$ be a family of subsets of $X$, containing $A$ and having properties $\left(\mathrm{b}^{\prime}\right)$ and $(\mathrm{e})$ above. Then $P$ contains the $\sigma$-field (of subsets of $X$ ) generated by $A$.

In fact, once the lemma is proved, we have only to apply it to $A=$ family of open subsets of $R \times 1$.

Proof of lemma. Let 2 be the smallest family of subsets of $X$ that contains $A$ and satisfies $\left(b^{\prime}\right)$ and (e) above; it suffices to prove that 2 is a $\sigma$-field. We first show:

$$
\text { If } A \in A \text { and } B \in Q \text { then } A \cap B \in Q \text {. }
$$

For put $C=$ family of all $B \in Q$ for which $(\alpha)$ is true (for all $A \in A$ ); one easily verifies that $C \supset A$ and satisfies $\left(b^{\prime}\right)$ and (e), so $C \supset 2$, as required.

By repeating the above argument with slight modifications, we deduce from ( $\alpha$ ) that:

$$
\text { If } A, B \in Q \text { then } A \cap B \in \mathcal{Q} \text {. }
$$

Hence

$$
\text { If } P, Q \in Q \text { then } Q-P \in Q \text { : }
$$

for we now know $Q \cap P \in Q$, and apply ( $\left(b^{\prime}\right)$ to $Q$ and $Q \cap P$.

It follows readily that $Q$ is closed under finite unions, and hence under countable (not necessarily disjoint) unions; and, being closed under complements, 2 is a $\sigma$-field.

\section{An explicit isometry for a special case.}

5.1. In this section we prove what amounts to a very special case of the main theorem, in which the compact measure space $S$ is topologically a subset 
of the real line. Suppose, then, that $S$ is a closed subset of the unit interval $I$ and that $\mu$ is a Borel measure on $S$ (necessarily regular) with $\mu(S)=1$; it is not assumed that $\mu$ is nonatomic. We consider the measure-theoretic product $(S \times H, \mu \times M)$ where $(H, M)$ is the measure-theoretic product of $\aleph_{0}$ copies of $I$, each with Lebesgue measure $m$. We shall give an explicit construction to prove:

THEOREM. There is a Borel isometry $\phi$ of $(S \times H, \mu \times M)$ onto $(H, M)$.

That is, $\phi$ is a bijection of $S \times H$ onto $H$ such that $\phi$ and $\phi^{-1}$ both take Borel sets to Borel sets, and such that, for every measurable $E \subset S \times H$, $M(\phi(E))=(\mu \times M)(E)$. As mentioned in the introduction, though the existence of such a $\phi$ is well known, we need an explicit construction of it for use in $\S 6$. The following construction may not be the simplest possible, but is in a form convenient for later work.

5.2. As in $\S 4$, it is convenient to extend $\mu$ to a Borel measure on all of $I$, by taking it to vanish outside $S$. For $t \in I$ define

$$
d(t)=\mu[0, t)=\mu(S \cap[0, t)) ;
$$

then $d$ is an increasing function from $I$ to $I$, and the considerations of $\S 2$ apply to it. Because $\mu(S)=1$ we have(7)

$$
d_{-}(t)=d(t), \quad d_{+}(t)=\mu[0, t] .
$$

(However, we shall sometimes write $d_{-}$rather than $d$, for the sake of notational symmetry.)

In the notation of $\S 2, J(d)$ is the set of atomic points of $(S, \mu)$; and, since $I-S$ is the union of a family of disjoint intervals, open relative to $I$, on each of which $d$ is constant, we have $I-S \subset C(d) \subset C^{\prime}(d)$. Thus

$$
J(d) \cup G(d) \subset S .
$$

From $\S 2$ we know that $d \mid G(d)$ is a strictly increasing (and homeomorphic) map of $G(d)$ onto $G\left(d^{+}\right)$. We shall show that $d \mid G(d)$ is also measure-preserving; that is,

$$
\mu(E)=m(d(E)) \text { for every Borel subset } E \text { of } G(d) .
$$

It is enough to prove (3) when $E$ is of the form $G(d) \cap[0, t], t \in I$. Now the definition of $G(d)(\S 2)$ can be written

$$
G(d)=(I-J(d))-\left(C^{\prime}(d)-J(d)\right)
$$

(7) Note that this fits the convention at the beginning of $\S 2$, which requires $d_{-}(0)=$ $0, d_{+}(1)=1$. 
and here we have

$$
\mu\left(C^{\prime}(d)-J(d)\right)=0,
$$

because by definition $(\S 2) C^{\prime}(d)$ is a union of intervals of the form $\left[a_{p}, b_{p}\right]$ $(p \in K(d))$ where $d$ is constant and equal to $p$ on the open interval $\left(a_{p}, b_{p}\right)$; thus

$$
C^{\prime}(d)-J(d)=\bigcup_{p \in K(d)}\left[a_{p}, b_{p}\right]^{\prime}
$$

where the prime denotes that any endpoints $\left(a_{p}\right.$ or $\left.b_{p}\right)$ that are in $J(d)$ are deleted. For later use we remark that here $a_{p}=d_{\leftarrow}(p)$ and $b_{p}=d^{\leftarrow}(p)$, from 2(13). Now all undeleted endpoints in (6) are $\mu$-null; thus (5) follows from (6) together with the observation that

$$
\mu\left(a_{p}, b_{p}\right)=d_{-}\left(b_{p}\right)-d_{+}\left(a_{p}\right)=p-p=0 .
$$

From (4) we now have, for $t \in I$,

$$
\mu(G(d) \cap[0, t])=d_{+}(t)-\sum\{\mu(s) \mid s \in J(d), s \leqslant t\} .
$$

Again, since $d(G(d))=G\left(d^{\leftarrow}\right)$ and $d \mid G(d)$ is strictly increasing, we have

$$
d(G(d) \cap[0, t])=G\left(d^{\leftarrow}\right) \cap\left[0, d_{+}(t)\right],
$$

where $G\left(d^{\leftarrow}\right)$ is by definition $\left(I-C^{\prime}\left(d^{\leftarrow}\right)\right)-J\left(d^{+}\right)$. The countable set $J\left(d^{\leftarrow}\right)$ is $m$-null; thus from (8) we obtain

$$
m(d(G(d) \cap[0, t]))=d_{+}(t)-m\left(C^{\prime}\left(d^{\leftarrow}\right) \cap\left[0, d_{+}(t)\right]\right) .
$$

Now 2(12) shows that $C^{\prime}\left(d^{+}\right)=\bigcup\left\{\left[d_{-}(s), d_{+}(s)\right] \mid s \in J(d)\right\}$; here we may delete the end points without altering $m$-measures, obtaining

$$
\begin{aligned}
m\left(C^{\prime}\left(d^{\leftarrow}\right) \cap\left[0, d_{+}(t)\right]\right) & =\sum\left\{d_{+}(s)-d_{-}(s) \mid s \in J(d), s \leqslant t\right\} \\
& =\sum\{\mu(s) \mid s \in J(d), s \leqslant t\} .
\end{aligned}
$$

With (9) and (7) this shows that (3) holds when $E=G(d) \cap[0, t]$; and hence it holds generally.

5.3. Now define a function $\delta$ from $S \times I$ to $I$ by:

$$
\delta(s, y)=(1-y) d_{-}(s)+y d_{+}(s) \quad(s \in S, y \in I) \text {. }
$$

Of course $\delta$ is $B$-measurable because $d_{-}$and $d_{+}$are (being semicontinuous). Clearly $\delta(s, 0)=d_{-}(s)=d(s), \delta(s, 1)=d_{+}(s)$, and $0 \leqslant d_{-}(s) \leqslant \delta(s, y) \leqslant$ $d_{+}(s) \leqslant 1$. It readily follows that

if $s, s^{\prime} \in S$ and $s<s^{\prime}$ then $\delta(s, y) \leqslant \delta\left(s^{\prime}, z\right)$

for all $y, z \in I$. 
We observe that

$$
\delta(S \times I)=I .
$$

For, from (1) and 2(13),

$$
\delta(S \times\{0\})=d_{-}(S)=d(I) \supset I-\bigcup\left\{\left[d_{-}(t), d_{+}(t)\right] \mid t \in J(d)\right\} ;
$$

and, for each $t \in J(d)$ we have $t \in S$ and

$$
\delta(\{t\} \times I)=\left[d_{-}(t), d_{+}(t)\right],
$$

so (11) follows.

Next we need a formula for $\delta^{-1}(p)$ where $p \in K(d)$. We recall that $d^{-1}(p)$ is an interval, with endpoints $a_{p}=d_{\leftarrow}(p), b_{p}=d^{\leftarrow}(p)$, and $a_{p}, b_{p} \in$ $S \cup\{0,1\}$; in fact $a_{p}$ can fail to be in $S$ only if it is 0 , and $b_{p}$ can fail to be in $S$ only if it is 1 . Now write

$$
\begin{array}{lll}
A_{p}=\{1\} \text { if } a_{p} \in J(d), & I \text { if } a_{p} \in S-J(d), & \varnothing \text { if } a_{p} \notin S ; \\
B_{p}=\{0\} \text { if } b_{p} \in J(d), & I \text { if } b_{p} \in S-J(d), & \varnothing \text { if } b_{p} \notin S .
\end{array}
$$

Then one verifies:

(12) If $p \in K(d), \delta^{-1}(p)=\left(\left(S \cap\left(a_{p}, b_{p}\right)\right) \times I\right) \cup\left(\left\{a_{p}\right\} \times A_{p}\right) \cup\left(\left\{b_{p}\right\} \times B_{p}\right)$.

Of course $\delta^{-1}(p)$ is Borel in $S \times I$ (and hence in $I \times I$ ) for each $p \in$ $K(d)$. Further, $\mu\left(a_{p}, b_{p}\right)=0$ and $\mu\left\{a_{p}\right\}=0$ if $a_{p} \notin J(d)$, and $\mu\left\{b_{p}\right\}=0$ if $b_{p} \notin J(d)$. So (12) shows that $(\mu \times m)\left(\delta^{-1}(p)\right)=0(p \in K(d))$; and, because $K(d)$ is countable, it follows that

$$
(\mu \times m)\left(\delta^{-1}(K(d))\right)=0 .
$$

5.4. Now consider the pseudo-inverse $d^{\leftarrow}$ of $d$; from $\S 2$, on defining

$$
I_{1}=G\left(d^{\leftarrow}\right), \quad I_{2}=J\left(d^{\leftarrow}\right), \quad I_{3}=C^{\prime}\left(d^{\leftarrow}\right)-J\left(d^{\leftarrow}\right),
$$

we have that $I_{1}, I_{2}, I_{3}$ are pairwise disjoint Borel sets with union $I$. Define $T_{i}=\delta^{-1}\left(I_{i}\right)(i=1,2,3)$; then $T_{1}, T_{2}, T_{3}$ are disjoint Borel sets with union $S \times I$. We shall show that

$$
T_{1}=G(d) \times I, \quad T_{2}=\delta^{-1}(K(d)), \quad T_{3}=(J(d) \times I)-T_{2} .
$$

If $(s, x) \in G(d) \times I$, then $d_{-}(s)=d(s)=d_{+}(s)$ and so $\delta(s, x)=d(s)$ $\in d(G(d))=G\left(d^{\leftarrow}\right)$, by $\S 2$. Conversely, if $\delta(s, x)=p$ (say) $\in G\left(d^{\leftarrow}\right)$, put $s_{0}=d_{\leftarrow}(p)=d^{\leftarrow}(p)$; then $s_{0} \in d^{\leftarrow}\left(G\left(d^{\leftarrow}\right)\right)=G(d)$, and we show $s=s_{0}$. If $s<s_{0}$ then $s<d_{\leftarrow}(p)$; hence $(2(5)) d_{+}(s)<p$, and it follows that $\delta(s, x)<p$, which is impossible. Similarly $s>s_{0}$ is impossible; hence $s=s_{0}$, and therefore $(s, x) \in G(d) \times I$. This verifies the first assertion in (14). 
Since $J\left(d^{-}\right)=K(d)(2(13))$, the second assertion is clear. The third is more troublesome. By the argument used to prove (6), but applied to $d^{\leftarrow}$ instead of $d$, we have

$$
I_{3}=\bigcup\left\{\left[d_{-}(t), d_{+}(t)\right]^{\prime} \mid t \in J(d)\right\},
$$

where the prime denotes the endpoints in $K(d)$, if any, are to be removed. Suppose now that $\delta(s, x) \in I_{3}$; then $\delta(s, x) \in\left[d_{-}(t), d_{+}(t)\right]^{\prime}$ for some $t \in J(d)$. It follows (as in proving the first assertion) that $s=t$; thus $(s, x) \in$ $J(d) \times I$ and since $I_{3}$ is disjoint from $I_{2}$ we also have $(s, x) \notin \delta^{-1}\left(I_{2}\right)=T_{2}$. Conversely, if $(s, x) \in(J(d) \times I)-T_{2}$, one easily checks that $\delta(s, x) \in C^{\prime}\left(d^{\leftarrow}\right)-$ $I_{2}=I_{3}$, completing the proof.

A similar calculation gives

$$
T_{3}=\bigcup\left\{\{s\} \times I_{s} \mid s \in J(d)\right\},
$$

where $I_{s}$ is $I$ with possibly one or both of its endpoints removed. More precisely, the endpoint 0 is removed if and only if $d_{-}(s) \in K(d)$, and the endpoint 1 is removed if and only if $d_{+}(s) \in K(d)$.

It follows easily that

$$
\delta \mid T_{3} \text { is a bijection of } T_{3} \text { onto } I_{3} \text {. }
$$

5.5. Now we define the map $\phi: S \times H \rightarrow H$ as follows. We regard $H$ as $I \times H^{\prime}$, where $H^{\prime}$ is a copy of $H$; thus we have to define $\phi$ on $S \times I$ $\times H^{\prime}$, which is the union of 3 disjoint Borel sets $T_{i} \times H^{\prime}(i=1,2,3)$. We define $\phi$ on $T_{1} \times H^{\prime}=G(d) \times I \times H^{\prime}$ by

$$
\phi\left(s, x_{1}, x_{2}, x_{3}, \cdots\right)=\left(d(s), x_{1}, x_{2}, \cdots\right) \in H
$$

(where $s \in G(d), x_{1} \in I$ and $\left(x_{2}, x_{3}, \cdots\right) \in H^{\prime}$ ).

To define $\phi$ on $T_{2} \times H^{\prime}$, we first note that, for each $p \in K(d)$, $\delta^{-1}(p) \times I$ is an uncountable separable absolute Borel set, so we can choose [2, p. 450] a Borel isomorphism (that is, a bijection preserving Borel sets both ways), say $\psi_{p}$, of $\delta^{-1}(p) \times I$ onto $I$. Now if $\left(s, x_{1}, x_{2}, \cdots\right) \in T_{2} \times H^{\prime}$ we have $\left(s, x_{1}\right) \in T_{2}=\delta^{-1}(K(d))$, so $\delta\left(s, x_{1}\right)=p$, say, $\in K(d)$. We define $\phi$ on $T_{2} \times H^{\prime}$ by:

$$
\begin{gathered}
\phi\left(s, x_{1}, x_{2}, x_{3}, \cdots\right)=\left(p, \psi_{p}\left(s, x_{1}, x_{2}\right), x_{3}, x_{4}, \cdots\right) \\
\text { where } p=\delta\left(s, x_{1}\right) .
\end{gathered}
$$

Finally, on $T_{3} \times H^{\prime}$, define

$$
\phi\left(s, x_{1}, x_{2}, x_{3}, \cdots\right)=\left(\delta\left(s, x_{1}\right), x_{2}, x_{3}, \cdots\right) .
$$

Note that in all three cases the first coordinate of $\phi\left(s, x_{1}, x_{2}, \cdots\right)$ is $\delta\left(s, x_{1}\right)$. 
Then $\phi$ is injective. For suppose $\phi\left(s, x_{1}, x_{2}, \cdots\right)=\phi\left(s^{\prime}, x_{1}^{\prime}, x_{2}^{\prime}, \cdots\right)$, where $s, s^{\prime} \in S$ and $x_{n}, x_{n}^{\prime} \in I$ for $n=1,2, \cdots$. Then the first coordinates $\delta\left(s, x_{1}\right)$ and $\delta\left(s^{\prime}, x_{1}^{\prime}\right)$ of these points are equal, and belong to some $I_{t}$ $(i=1,2,3)$. By definition of $T_{i}$, it follows that both $\left(s, x_{1}\right)$ and $\left(s^{\prime}, x_{1}^{\prime}\right)$ are in $T_{i}$. If $i=1$ here, (17) shows that $d(s)=d\left(s^{\prime}\right), x_{1}=x_{1}^{\prime}, x_{2}=x_{2}^{\prime}, \cdots$. Also $s, s^{\prime} \in G(d)$ (by (14)), on which $d$ is injective; thus $s=s^{\prime}$, as required. If $i=2$ we again have $\delta\left(s, x_{1}\right)=\delta\left(s^{\prime}, x_{1}^{\prime}\right)=p$, say, and (18) gives $x_{3}=x_{3}^{\prime}$, $x_{4}=x_{4}^{\prime}, \cdots$, and $\psi_{p}\left(s, x_{1}, x_{2}\right)=\psi_{p}\left(s^{\prime}, x_{1}^{\prime}, x_{2}^{\prime}\right)$. Since $\psi$ is injective we have $s=s^{\prime}, x_{1}=x_{1}^{\prime}$ and $x_{2}=x_{2}^{\prime}$ also, again as required. Finally if $i=3$ the result follows in a similar way from (19) and (16).

Also $\phi$ is surjective. Given $y=\left(y_{1}, y_{2}, \cdots\right) \in H$, we have $y_{1} \in I_{i}$ for some $i=1,2,3$. If $i=1$, then $\varnothing \neq \delta^{-1}\left(y_{1}\right) \subset G(d) \times I$, from (11) and (14), so there exist $s \in G(d)$ and $x \in I$ such that $(s, x)=y_{1}$. Since $d$ is continuous at each point of $G(d), d(s)=\delta(s, x)=y_{1}$. Thus $\phi\left(s, y_{2}, y_{3}, \cdots\right)$ $=\left(y_{1}, y_{2}, y_{3}, \cdots\right)$ as required. The arguments in the cases $i=2,3$ are similar. Incidentally this also proves

$$
\phi\left(T_{i} \times H^{\prime}\right)=I_{i} \times H^{\prime} \quad(i=1,2,3) .
$$

5.6. Next we show that, for each Borel set $E$ in $S \times H, \phi(E)$ is a Borel set in $H$. It suffices to prove this when $E \subset T_{i} \times H^{\prime}, i=1,2,3$. If $i=1$ here, we note that (17) can be written $\phi \mid T_{1} \times H^{\prime}=(d \mid G(d)) \times i_{H}$ where $i_{H}$ is the identity map of $H$, and this is a homeomorphism. Thus $\phi(E)$ is Borel in $\phi\left(T_{1} \times H^{\prime}\right)=I_{1} \times H^{\prime}$, and hence is Borel in $H$. If $i=2$, we note that (from (14)) $T_{2}=\bigcup\left\{\delta^{-1}(p) \mid p \in K(d)\right\}$, a countable union; hence it is enough to consider the case in which $E$ is contained in a single set $\delta^{-1}(p) \times H^{\prime}$ $(p \in K(d))$. But on $\delta^{-1}(p) \times H^{\prime}$, from (18), $\phi$ is of the form $\alpha \times \psi_{p} \times i^{\prime}$, where $\alpha$ is a constant map, $\psi_{p}$ is a Borel isomorphism and $i^{\prime}$ is an identity map. Thus again $\phi(E)$ is Borel in $\phi\left(\delta^{-1}(p) \times H^{\prime}\right)$, which is itself Borel in $H$ (being the set of all points of $H$ with first coordinate $p$ ). Finally, if $i=3$, we use (15) to reduce the problem to the case in which $E \subset\{s\} \times I_{s} \times H^{\prime}$ for some $s \in J(d)$. On this set, $\phi$ reduces to $\delta \times i_{H^{\prime}}$, where $\delta \mid\{s\} \times I$ is linear; hence again $\phi(E)$ is Borel.

In other words, $\phi^{-1}$ is a Borel measurable bijection between separable absolute Borel sets; and from this it follows [2, p. 489] that $\phi$ is a Borel isomorphism. (Alternatively, it is easy to verify directly that $\phi$ is Borel measurable, by arguments similar to the preceding.)

5.7. To conclude the proof of the theorem, we have only to show that $\phi$ is measure-preserving; that is, if $E$ is a Borel subset of $S \times H$ then $M(\phi(E))=$ $(\mu \times M)(E)$. Again it suffices to do this assuming $E \subset T_{i} \times H^{\prime}, i=1,2,3$.

For $i=1$ we have as above that $\phi$ reduces to $(d \mid G(d)) \times i_{H}$, where 
both $i_{H}$ and (by (3) above) $d \mid G(d)$ are measure-preserving.

For $i=2$ we note that $(\mu \times M)\left(T_{2} \times H^{\prime}\right)=0$, from (13), and $\phi\left(T_{2} \times H^{\prime}\right)=J(d) \times H^{\prime}$ which is $M$-null because $J(d)$ is $m$-null (being countable). Thus $M(\phi(E))=0=(\mu \times M)(E)$ in this case.

Finally, for $i=3$, we may suppose as before that $E \subset\{s\} \times I_{s} \times H^{\prime}$, where $s \in J(d)$; and it is then enough to show that $\delta \mid\{s\} \times I_{s}$ is measure-preserving. Now if $y \in I$ we have that the increasing linear function $\delta$ on $\{s\} \times I$ maps the interval $\{s\} \times(0, y)$ onto the interval $\left(d_{-}(s),(1-y) d_{-}(s)+y d_{+}(s)\right)$, with $m$-measure $y\left(d_{+}(s)-d_{-}(s)\right)=y \mu(s)$, so $\delta$ here preserves measure for intervals, and thus for Borel sets also; and the proof is complete.

6. A more general isometry.

6.1. In the present section we deduce from the result just obtained a generalization of it which will be the essential step in the transfinite process leading to the main theorem. We suppose now that $R$ is a compact Hausdorff space and that $S$ is a closed (hence compact) subspace of $R \times I$ such that $\pi(S)=$ $R$, where $\pi$ is the projection map of $R \times I$ onto $R$. We use the same notation as in \$4; in particular, for $P \subset R \times I$ and $r \in R$, we write $P_{r}=\{t \in$ $I \mid(r, t) \in P\}$, and the restriction $\pi \mid S$ is denoted by $\theta$. As in $\S 4$, we suppose that a completed regular Borel measure $\mu$ is given on $S$, with $\mu(S)=1$; and we use $\lambda$ to denote the corresponding completed Borel measure on $R$ (that is, $\lambda=\mu \circ \theta^{-1}$ ). We recall that an isometry between two measure spaces is a bijection between them that preserves measurability and measure (both ways).

THEOREM. Under the above hypotheses, there exists an isometry $\Phi$ of $(S \times H, \mu \times M)$ onto $(R \times H, \lambda \times M)$ that "preserves r-fibres"; that is, the following diagram commutes, where $\omega, \omega$ are the projection maps:

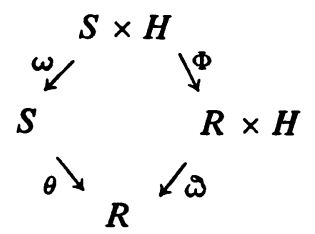

Of course, when $R$ reduces to a singleton, the present theorem reduces to the previous one.

6.2. Proof. Apply the disintegration theorem of 4.1 to obtain a Borel measure $\mu_{r}$ on each $S_{r}(r \in R)$ with the properties stated in that theorem. For each $r \in R$, apply the theorem of 5.1 to the measure space $\left(S_{r}, \mu_{r}\right)$ (of course $S_{r}$ is a closed nonempty subset of $I$ ) to obtain a Borel isometry $\phi_{r}$ of $\left(S_{r} \times H, \mu_{r} \times M\right)$ onto $(H, M)$, constructed as in $\S 5$. Define a map $\Phi$ : $S \times H \rightarrow R \times H$ by 


$$
\Phi(r, t, y)=\left(r, \phi_{r}(t, y)\right) \quad\left(r \in R, t \in S_{r}, y \in H\right) .
$$

It is easy to see that $\Phi$ is a bijection of $S \times H$ onto $R \times H$ that preserves $r$-fibres. We next show that $\Phi$ and $\Phi^{-1}$ preserve measurability; this is the only troublesome part of the proof.

6.3. It is convenient to extend $\mu_{r}$ to a measure (still denoted by $\mu_{r}$ ) on $I$, by taking $\mu_{r}$ to vanish outside $S_{r}(r \in R)$. Define functions $D_{-}, D_{+}$from $R \times I$ to $I$ by

$$
D_{-}(r, t)=\mu_{r}([0, t)), \quad D_{+}(r, t)=\mu_{r}([0, t]) \quad(t \in I) ;
$$

and for convenience write $D_{-}$also as $D$. Of course $0 \leqslant D=D_{-} \leqslant D_{+} \leqslant 1$. Note that, for fixed $r \in R$, the function $D_{r}: I \rightarrow I$, defined by $D_{r}(t)=$ $D(r, t)$, is precisely the function denoted in $\S 5$ (applied to $S_{r}$ ) by $d$; and similarly $D_{+r}$ (where $D_{+r}(t)=D_{+}(r, t)$ ) corresponds to $d_{+}$in $\S 5$, and $D_{-r}$ to $d_{-}$. In particular, $D_{r}=D_{-r}$ and is continuous on the left, for each $r \in R$.

Define $\Delta: R \times I \times I \rightarrow I$ by

$$
\Delta(r, t, y)=(1-y) D_{-}(r, t)+y D_{+}(r, t)
$$

(where $r \in R$, and $t, y \in I$ ); again for fixed $r$ this reduces to the function $\delta$ of 5.3. More precisely, if $\Delta_{r}(t, y)=\Delta(r, t, y)$, then $\delta$ corresponds to the restriction $\Delta_{r} \mid S_{r}$.

We observe that these functions are Borel measurable. In fact, for each fixed $t, D_{+}(r, t)$ is a Borel measurable function of $r$, because $D_{+}(r, t)=$ $\mu_{r}\left(P_{r}\right)$ where $P=(R \times[0, t]) \cap S$, and the Borel measurability is guaranteed by the disintegration theorem (4.1). And for fixed $r, D_{+}(r, t)$ is an increasing function of $t$, continuous on the right. So the theorem of 3.2 shows that $D_{+}$ is Borel measurable; and similar arguments apply to $D_{-}=D$ and to $\Delta$.

Thus, on defining $\widetilde{\Delta}: R \times I \times I \rightarrow R \times I$ by

$$
\widetilde{\Delta}(r, t, y)=(r, \Delta(r, t, y)) \quad(r \in R ; t, y \in I),
$$

we see from 3.1 that $\tilde{\Delta}$ is also Borel measurable.

6.4. Now for fixed $r \in R$ we recall that $\phi_{r}$ was defined (in $\S 5$, applied to $\left.\left(S_{r}, \mu_{r}\right)\right)$ by means of a decomposition of $I$ into 3 disjoint sets $I_{1}, I_{2}, I_{3}$ and a corresponding decomposition of $S_{r} \times I$ into sets $T_{1}, T_{2}, T_{3}$ (5.4). We now rename these sets $I_{r i}, T_{r i}(i=1,2,3)$; thus $T_{r i}=\delta_{r}^{-1}\left(I_{r i}\right)$ where $\delta_{r}$ (called $\delta$ in $\S 5$ ) is $\Delta_{r}$ restricted to $S_{r}$. Now define

$$
\begin{aligned}
& U_{i}=\left\{\{r\} \times T_{r i} \mid r \in R\right\} \quad(i=1,2,3) \quad \text { and } \\
& L_{i}=\left\{\{r\} \times I_{r i} \mid r \in R\right\} \quad(i=1,2,3)
\end{aligned}
$$

thus $L_{1}, L_{2}, L_{3}$ are disjoint sets with union $R \times I ; U_{1}, U_{2}, U_{3}$ are disjoint 
sets with union $S \times I$, and $U_{i}=(\tilde{\Delta} \mid S \times I)^{-1}\left(L_{i}\right)$. From $5(16)$ we see that $\widetilde{\Delta} \mid U_{3}$ is a bijection of $U_{3}$ onto $L_{3}$. On using $5(14)$ and the definitions of the sets $I_{r i}$, we see that (in the notation of 3.4)

$$
\begin{array}{lll}
L_{1}=G^{*}\left(D^{\leftarrow}\right), & L_{2}=J^{*}\left(D^{\leftarrow}\right), & L_{3}=C^{*}\left(D^{\leftarrow}\right)-J^{*}\left(D^{\leftarrow}\right), \\
U_{1}=G^{*}(D) \times I, & U_{2}=(S \times I) \cap \tilde{\Delta}^{-1}\left(K^{*}(D)\right), & U_{3}=\left(J^{*}(D) \times I\right)-U_{2} .
\end{array}
$$

From 3.5 these sets are Borel subsets of $R \times I$ and $S \times I$ respectively. Further, from 5.(20), $\Phi$ maps each $U_{i} \times H^{\prime}$ onto $L_{i} \times H^{\prime}(i=1,2,3)$, where $H=I \times H^{\prime}$.

We show that $\Phi$ and $\Phi^{-1}$ are measurable by showing that the restrictions of $\Phi$ to $U_{1} \times H^{\prime}$ and $U_{3} \times H^{\prime}$ are Borel isomorphisms, and that $U_{2} \times H^{\prime}$ and $L_{2} \times H^{\prime}$ are both null. For $U_{1} \times H^{\prime}$ this is simple; the restriction of $\Phi$ to this set coincides with $\widetilde{D} \times i_{H}$, where $i_{H}$ is the identity map of $H$ and $\widetilde{D}: G^{*}(D)$ $\rightarrow G^{*}\left(D^{\leftarrow}\right)$ is a bijection, with inverse $\left(D^{\leftarrow}\right)^{\sim}$. From $\S 3$, both $\widetilde{D}$ and $\left(D^{\leftarrow}\right)^{\sim}$ are Borel measurable; hence $\Phi \mid U_{1} \times H^{\prime}$ is a Borel isomorphism onto $G^{*}\left(D^{\leftarrow}\right)$ $\times H^{\prime}=L_{1} \times H^{\prime}$, as desired.

6.5. On $U_{3} \times H^{\prime}$ the situation is less simple. It is easily seen that the restriction of $\Phi$ to $U_{3} \times H^{\prime}$ coincides with $\widetilde{\Delta} \times i_{H^{\prime}}$, so it is enough to verify that $\widetilde{\Delta} \mid U_{3}$ is a Borel isomorphism. We have already seen that $\widetilde{\Delta}$ is Borel measurable, so we have only to show that $\widetilde{\Delta}(E)$ is Borel in $R \times I$ for every Borel set $E \subset U_{3}$. Now one verifies via 5(15) that $U_{3}=V_{1} \cup V_{2} \cup V_{3}$ where

$$
\begin{aligned}
& V_{1}=J^{*}(D) \times(0,1), \\
& V_{2}=\left\{(r, t, 0) \mid r \in R, t \in J\left(D_{r}\right), D_{-}(r, t) \notin K\left(D_{r}\right)\right\}, \\
& V_{3}=\left\{(r, t, 1) \mid r \in R, t \in J\left(D_{r}\right), D_{+}(r, t) \notin K\left(D_{r}\right)\right\} ;
\end{aligned}
$$

and these 3 sets are Borel because they are the intersections of $U_{3}$ with $S \times(0,1), S \times\{0\}, S \times\{1\}$ respectively. So it is enough to deal with the 3 cases $E \subset V_{i}, i=1,2,3$, separately.

First suppose $E \subset V_{1}$, and that $E$ is of the very special form $E=$ $J^{*}(D) \times(0, z)$, where $0<z \leqslant 1$, Let $\rho$ be a rational number in $(0,1)$. Keeping $\rho$ and $z$ fixed for the present, write

$$
\begin{aligned}
t_{r} & =D^{\leftarrow}(r, \rho), \quad a_{r}=D_{-}\left(r, t_{r}\right)=D\left(r, t_{r}\right), \\
b_{r} & =(1-z) D_{-}\left(r, t_{r}\right)+z D_{+}\left(r, t_{r}\right), \\
H_{\rho z} & =\left\{(r, p) \mid r \in R, a_{r}<p<b_{r}\right\} .
\end{aligned}
$$

Then $t_{r}, a_{r}, b_{r}$ are Borel measurable functions of $r$ (because $D, D_{-}$and $D_{+}$ are Borel measurable); thus, from 3.6, $H_{\rho z}$ is a Borel subset of $R \times I$. Now, letting $\rho$ vary, one readily verifies that 


$$
\widetilde{\Delta}\left(J^{*}(D) \times(0, z)\right)=\bigcup_{\rho} H_{\rho z},
$$

and is therefore a Borel set, as required.

In particular, on taking $z=1$, we see that $\widetilde{\Delta}\left(V_{1}\right)$ is Borel in $R \times I$.

Next suppose that the subset $E$ of $V_{1}$ is of the form $E=A \times(0,1)$, where $A \in B\left(J^{*}(D)\right)$. It can be checked that in this case $\widetilde{\Delta}(E)=\widetilde{\Delta}\left(V_{1}\right) \cap$ $\left(\left(D^{\leftarrow}\right)^{\sim}\right)^{-1}(A)$, which again is Borel in $R \times I$. Thus $\widetilde{\Delta}(E)$ is Borel whenever $E$ belongs to the $\sigma$-field of subsets of $V_{1}=J^{*}(D) \times(0,1)$ generated by the sets $J^{*}(D) \times(0, z), A \times(0,1)\left(0<z<1, A \in B\left(J^{*}(D)\right)\right)$; that is, whenever $E$ is Borel in $V_{1}$.

It is not essential for present purposes to verify that $\widetilde{\Delta}(E)$ is also Borel for Borel subsets $E$ of $V_{2}$ or $V_{3}$, because it is not hard to see that both $V_{2} \cup V_{3}$ and $\widetilde{\Delta}\left(V_{2} \cup V_{3}\right)$ are null sets. But this fact (that $\widetilde{\Delta} \mid V_{2}$ and $\widetilde{\Delta} \mid V_{3}$ preserve Borel sets) will be needed later; it can be shown as follows. We have $V_{2}=W_{2} \times\{0\}$ where

$$
W_{2}=\left\{(r, t) \in J^{*}(D) \mid D(r, t) \notin K\left(D_{r}\right)\right\} ;
$$

and for $(r, t) \in W_{2}$ we have $\widetilde{\Delta}(r, t, 0)=(r, D(r, t))=\widetilde{D}(r, t)$. Thus to deal with $\widetilde{\Delta} \mid V_{2}$ it is enough to show that $\widetilde{D} \mid W_{2}$ preserves Borel sets. Now, for each rational $\rho$ in $(0,1)$, write

$$
X_{\rho}=\bigcup\left\{\{r\} \times D\left(r, D^{\leftarrow}(r, \rho)\right) \mid r \in R, D^{\leftarrow}(r, \rho) \in J\left(D_{r}\right)\right\} ;
$$

we first show that $X_{\rho}$ is Borel ( in $R \times I$ ). To do this, keeping $\rho$ fixed, we put $t_{r}=D^{\leftarrow}(r, \rho), a_{r}=D\left(r, t_{r}\right), B=\left\{r \in R \mid t_{r} \in J\left(D_{r}\right)\right\}, A=\left\{\left(r, a_{r}\right) \mid r \in R\right\}$. As before, $t_{r}$ and $a_{r}$ are Borel measurable functions of $r$, and therefore $A$ and $B$ are Borel sets in $R \times I, R$ respectively. But it is easy to see that $X_{\rho}=A \cap(B \times I)$, a Borel set as asserted.

Now we let $\rho$ vary, and put $Q=\bigcup_{\rho} X_{\rho}-K^{*}(D)$; this is also a Borel subset of $R \times I$. A straightforward (though tedious) verification will check that $\widetilde{D} \mid W_{2}$ is a bijection of $W_{2}$ onto $Q$, with inverse map $\left(D^{\leftarrow}\right)^{\sim} \mid Q$. Since $\left(D^{\leftarrow}\right)^{\sim}$ is known to be Borel measurable, it follows that $\widetilde{D}(E)$ is Borel for every Borel $E \subset W_{2}$. This deals with $V_{2}$, and the argument for $V_{3}$ is similar.

6.6. We have now checked that $\Phi$, restricted to $\left(U_{1} \cup U_{3}\right) \times H^{\prime}$, is a Borel isomorphism (onto $\left.\left(L_{1} \cup L_{3}\right) \times H^{\prime}\right)$; we next show that this restriction is measure-preserving. Suppose $E$ is a Borel subset of $\left(U_{1} \cup U_{3}\right) \times H^{\prime}$; then $\Phi(E)$ is a Borel subset of $R \times H$, and by Fubini's theorem its measure is

where

$$
(\lambda \times M)(\Phi(E))=\int_{R} M\left(\Phi(E)_{r}\right) d \lambda(r),
$$

$$
\Phi(E)_{r}=\{h \in H \mid(r, h) \in \Phi(E)\}=\phi_{r}\left(E_{r}\right)
$$

where 


$$
E_{r}=\{(t, h) \in I \times H \mid(r, t, h) \in E\} .
$$

Since $E_{r} \subset S_{r} \times H$, the fact that $\phi_{r}$ is an isometry (of $\left(S_{r} \times H, \mu_{r} \times M\right)$ onto $(H, M))$ gives

$$
(\lambda \times M)(\Phi(E))=\int_{R}\left(\mu_{r} \times M\right)\left(E_{r}\right) d \lambda(r),
$$

which by Fubini's theorem again (all sets arising here being Borel) equals

$$
\int_{H}\left\{\int_{R} \mu_{r}\left(E_{r h}\right) d \lambda(r)\right\} d M(h),
$$

where

$$
E_{r h}=\{t \in I \mid(r, t, h) \in E\} .
$$

Thus, from the properties of $\mu_{r}$ provided by the theorem of 4.1 , we have

$$
(\lambda \times M)(\Phi(E))=\int_{H} \mu\left(E_{h}\right) d M(h),
$$

where

$$
E_{h}=\{(r, t) \in R \times I \mid(r, t, h) \in E\} \subset S ;
$$

and so, finally, $(\lambda \times M)(\Phi(E))=(\mu \times M)(E)$, as required.

6.7. Finally, to show that $\Phi$ and $\Phi^{-1}$ (unrestricted) preserve measurability and measure, it now suffices to verify that both $U_{2} \times H^{\prime}$ and $\Phi\left(U_{2} \times H^{\prime}\right)$ are null. Now the same reasoning as in 6.6, applied to $E=U_{2} \times H^{\prime}$, gives

$$
(\lambda \times M)\left(\Phi\left(U_{2} \times H^{\prime}\right)\right)=(\mu \times M)\left(U_{2} \times H^{\prime}\right) .
$$

But $\Phi\left(U_{2} \times H^{\prime}\right)=L_{2} \times H^{\prime}=J^{*}\left(D^{\leftarrow}\right) \times H^{\prime}$; so, for fixed $r \in R$ and $h^{\prime} \in H^{\prime}$ the section $\left\{t \in I \mid\left(r, t, h^{\prime}\right) \in \Phi\left(U_{2} \times H^{\prime}\right)\right\}$ is just $J\left(D_{r}^{\leftarrow}\right)$, which is countable and therefore $m$-null. Thus one last application of Fubini's theorem shows $(\lambda \times M)\left(\Phi\left(U_{2} \times H^{\prime}\right)\right)=(\mu \times M)\left(U_{2} \times H^{\prime}\right)=0$, and the proof is complete.

6.8. For later use, we note the following sharpening of the above theorem for the special case in which $S=R \times I$. (It would be interesting to know whether it is valid in general.)

THEOREM. If $S=R \times I$ in the theorem of 6.1, then the isometry $\Phi$ can be taken to be a Borel isometry.

That is, we can arrange that, in addition to the properties of $\Phi$ specified in the theorem of 6.1, $\Phi$ and $\Phi^{-1}$ also take Borel sets to Borel sets. This has already been verified for the restrictions of $\Phi$ to $U_{1} \times H^{\prime}$ and $U_{3} \times H^{\prime}$ (and their inverses), so it is enough to deal with $\Phi$ on $U_{2} \times H^{\prime}$. We have seen that both $U_{2} \times H^{\prime}$ and its image $L_{2} \times H^{\prime}$ are null sets, so all that remains is to adjust $\Phi$ on $U_{2} \times H^{\prime}$ so that it becomes a Borel isomorphism of $U_{2} \times H^{\prime}$ onto $L_{2} \times H^{\prime}$. To do this, recall (6.4) that 


$$
U_{2}=\bigcup\left\{\{r\} \times T_{r 2} \mid r \in R\right\} \text { where } T_{r 2}=\delta_{r}^{-1}\left(I_{r 2}\right) ;
$$

$L_{2}=J^{*}\left(D^{\leftarrow}\right)=K^{*}(D)$; and (6.2) $\Phi(r, t, x)=\left(r, \phi_{r}(t, x)\right)$ where, for $(t, x) \in$ $T_{2 r} \times H^{\prime}$, we have (from 5(18)) $\phi_{r}(t, x)=\left(p, \psi_{p r}\left(t, x_{1}, x_{2}\right), x_{3}, \cdots\right)$ where

$$
p=\Delta\left(r, t, x_{1}\right)=\left(1-x_{1}\right) D_{-}(r, t)+x_{1} D_{+}(r, t) \in K\left(D_{r}\right)=J\left(D_{r}^{\leftarrow}\right)=I_{r 2},
$$

and where $\psi_{p r}$ is a Borel isomorphism of $\delta_{r}^{-1}(p) \times I$ onto $I$. So far, $\psi_{p r}$ has been chosen arbitrarily and independently for each $r$ (and $p$ ); and this is basically why $\Phi$ at present need not be a Borel isomorphism. Now, using the fact that here $S=R \times I$, we shall specify the maps $\psi_{p r}$ in a more uniform way.

For fixed $r \in R$ we have (from 5(12) applied to $S_{r}$, which now $=I$ ) that, for $p \in I_{r 2}=J\left(D_{r}^{\leftarrow}\right)=K\left(D_{r}\right)$,

$$
\delta_{r}^{-1}(p)=\left(\left(a_{p}, b_{p}\right) \times I\right) \cup\left(\left\{a_{p}\right\} \times A_{p}\right) \cup\left(\left\{b_{p}\right\} \times B_{p}\right)
$$

where $a_{p}=D_{r-}(p), b_{p}=D_{r}^{\leftarrow}(p)$ and $A_{p}=\{1\}$ if $a_{p} \in J\left(D_{r}\right), I$ otherwise; $B_{p}=\{0\}$ if $b_{p} \in J\left(D_{r}\right), I$ otherwise.

Note that $\delta_{r}^{-1}(p)$ consists of the rectangle $\left(a_{p}, b_{p}\right) \times I$ together with part of its boundary, and that there are 4 cases, depending on whether or not $J\left(D_{r}\right)$ contains $a_{p}$ and/or $b_{p}$. Corresponding to these 4 cases, we define

$$
\begin{array}{cc}
Y_{1}=K^{*}(D) \cap E \cap F, & Y_{2}=\left(K^{*}(D) \cap E\right)-F, \\
Y_{3}=\left(K^{*}(D)-E\right) \cap F, & Y_{4}=K^{*}(D)-(E \cup F),
\end{array}
$$

where $E=\left(\left(D_{\leftarrow}\right)^{\sim}\right)^{-1}\left(J^{*}(D)\right)$ and $F=\left(\left(D^{\leftarrow}\right)^{\sim}\right)^{-1}\left(J^{*}(D)\right)$. Thus $Y_{1}, Y_{2}$, $Y_{3}, Y_{4}$ are disjoint Borel sets with union $K^{*}(D)=L_{2}$. On writing $X_{i}=$ $(\tilde{\Delta})^{-1}\left(Y_{i}\right)(i=1,2,3,4)$ we have that $X_{1}, X_{2}, X_{3}, X_{4}$ are disjoint Borel sets with union $(\widetilde{\Delta})^{-1}\left(L_{2}\right)=U_{2}$.

Corresponding to the four kinds of rectangles arising above, we consider four standard rectangles:

$Z_{1}=(0,1) \times I$ together with the vertices $(0,1)$ and $(1,0)$;

$Z_{2}=(0,1) \times I$ together with the vertex $(0,1)$ and the edge $\{1\} \times I$;

$Z_{3}=(0,1) \times I$ together with the vertex $(1,0)$ and the edge $\{0\} \times I$; and $Z_{4}=I \times I$.

The product of each $Z_{t}$ with $I$ is Borel isomorphic to $I$, and we choose a fixed Borel isomorphism $\psi^{i}$ of $Z_{i} \times I$ onto $I(i=1,2,3,4)$. We now specify $\psi_{p r}$ to be the specific Borel isomorphism (of $\delta_{r}^{-1}(p) \times I$ onto $I$ ) obtained by scaling the appropriate $\psi^{i}$ appropriately; that is, 


$$
\psi_{p r}\left(t, x_{1}, x_{2}\right)=\psi^{i}\left(\left(t-a_{p}\right) /\left(b_{p}-a_{p}\right), x_{1}, x_{2}\right)
$$

where $(r, p) \in Y_{i}(i=1,2,3,4),\left(t, x_{1}\right) \in \delta_{r}^{-1}(p)$ and, as above, $a_{p}=$ $D_{\leftarrow}(r, p)$ and $b_{p}=D^{\leftarrow}(r, p)$. (Of course $b_{p}>a_{p}$ because $p \in J\left(D_{r}^{\leftarrow}\right)$ ).

The effect is to specify $\Phi$ on $U_{2} \times H^{\prime}=\bigcup_{i=1}^{4} X_{i} \times H^{\prime}$ as follows: For $(r, t) \in X_{i}$ and $\left(x_{1}, x_{2}, \cdots\right) \in H^{\prime}, \Phi\left(r, t, x_{1}, x_{2}, \cdots\right)=\left(r, p, q, x_{3}\right.$, $\left.x_{4}, \cdots\right)$ where $(r, p)=\widetilde{\Delta}\left(r, t, x_{1}\right)$ (that is, $p=\Delta\left(r, t, x_{1}\right)$ ) and

$$
q=\psi^{i}\left(\left(t-D_{\leftarrow}(r, p)\right) /\left(D^{\leftarrow}(r, p)-D_{\leftarrow}(r, p)\right)\right) .
$$

Thus, as a composition of Borel measurable functions, $\Phi$ is now Borel measurable on each $X_{i} \times H^{\prime}$, and so on $U_{2} \times H^{\prime}$.

A routine calculation shows that the inverse map $\Phi^{-1}$ on $L_{2} \times H^{\prime}=$ $\bigcup_{i=1}^{4} Y_{i} \times H^{\prime}$ is now given by: For $(r, p) \in Y_{i}$ and $\left(y_{2}, y_{3}, \cdots\right) \in H^{\prime}$, $\Phi^{-1}\left(r, p, y_{2}, y_{3}, \cdots\right)=\left(r, x_{1}, x_{2}, x_{3}, y_{3}, y_{4}, \cdots\right)$ where

$$
\begin{aligned}
& x_{1}=\left(1-\pi_{1}\left(\left(\psi^{i}\right)^{-1} y_{2}\right)\right) D_{\leftarrow}\left(r, y_{1}\right)+\pi_{1}\left(\left(\psi^{i}\right)^{-1} y_{2}\right) D^{\leftarrow}\left(r, y_{1}\right), \\
& x_{2}=\pi_{1}\left(\left(\psi^{i}\right)^{-1}\left(y_{2}\right)\right), \quad x_{3}=\pi_{3}\left(\left(\psi^{i}\right)^{-1}\left(y_{2}\right)\right),
\end{aligned}
$$

where $\pi_{1}, \pi_{2}, \pi_{3}$ denote the projection maps of the unit cube $I \times I \times I$ onto its respective coordinate spaces. Thus $\Phi^{-1}$ is also Borel measurable, and the proof is complete.

\section{Some properties of pseudo-isometries.}

7.1. The definition of a pseudo-isometry, from one measure space to another, was given in $\S 1$; as remarked there, it can be rephrased as follows (with a further slight change of notation). A pseudo-isometry $\phi$ from $(X, B, \mu)$ to $\left(X^{\prime}, B^{\prime}, \mu^{\prime}\right)$ is just an isometry (= point-isometry) from $(X, C, \mu)$ to $\left(X^{\prime}, B^{\prime}, \mu^{\prime}\right)$, where $C$ is a $\sigma$-subfield of $B$ that is "full" in the sense of including at least one member of each measure class. From this it is easy to see that the composition of two pseudo-isometries is a pseudo-isometry.

7.2. In this paragraph, all measures considered are to be finite and complete with respect to null sets. We first note some familiar measure-theoretic facts.

$$
\text { Suppose } \phi_{i}:\left(X_{i}, B_{i}, \mu_{i}\right) \rightarrow\left(X_{i}^{\prime}, B_{i}^{\prime}, \mu_{i}^{\prime}\right) \text { is }
$$
such that $\phi_{i}^{-1}$ preserves measurability and measure $(i=1,2)$; then the same is true of $\left(\phi_{1} \times \phi_{2}\right)^{-1}$.

Here, of course, $\phi_{1} \times \phi_{2}$ denotes the usual product map of $X_{1} \times X_{2}$ in $X_{1}^{\prime} \times X_{2}^{\prime}$, and these produce spaces are given the product measures.

$$
\text { Consequently the Cartesian product of two isometries }
$$
is an isometry. 
Also it is easy to show by standard methods that:

If $C_{i}$ is a full $\sigma$-subfield of $B_{i}$ in (1) $(i=1,2)$,

then $C_{1} \times C_{2}$ is a full $\sigma$-subfield of $B_{1} \times B_{2}$.

Here, as in 3.1, $C_{1} \times C_{2}$ and $B_{1} \times B_{2}$ denote the usual product $\sigma$-fields. It follows immediately that

The Cartesian product of two pseudo-isometries is a pseudo-isometry.

REMARK. All these results extend to products of arbitrarily many factors (assuming each of them has measure 1), but we shall not need this.

7.3. The following result about extending pseudo-isometries will play an essential part in the next section. (It will also be generalized later, in $\S 10$.) We suppose, as in $\S \S 4$ and 6 , that $R$ is a compact Hausdorff space, $S$ is a closed subset of $R \times I$ such that $\pi(S)=R$, where $\pi: R \times I \rightarrow R$ is the projection map; further, $\mu$ is a completed finite regular Borel measure on $S$, and $\lambda$ is the corresponding measure on $R$. Let $A$ be an arbitrary (nonempty) index set, and let $H^{A}$ denote the measure-theoretic product $\Pi\left\{H_{\alpha} \mid \alpha \in A\right\}$, where each $H_{\alpha}$ is a copy of $H$; we give $H^{A}$ the usual product measure $M^{A}$. It should be observed that, although $M^{A}$ is usually defined as a completed Baire measure, it can be equally regarded as a completed Borel measure because all open subsets of $H^{A}$ are $M^{A}$-measurable (see [6, Corollary to Theorem 4, p. 993] for the equivalent statement about $\left.\left(I^{A}, m^{A}\right)\right)$. In the following theorem it is understood that all product spaces have the usual product measures (completed with respect to null sets), the factors $R, S, H$ having measures $\lambda, \mu, M$ respectively.

THEOREM. Given a pseudo-isometry $\psi$ of $R \times H^{A}$ onto $H^{A}$, there exists a pseudo-isometry $\Theta$ of $S \times H^{A} \times H$ onto $H^{A} \times H$ making the following diagram commute, where $\omega$ and $\omega$ are the projection maps.

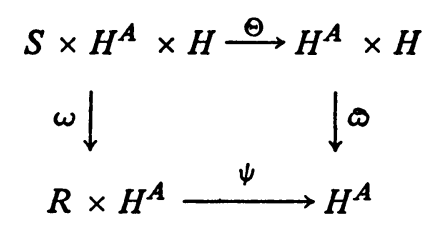

(The map $\omega$ is perhaps not self-explanatory; it is given by $\omega(s, x, y)=$ $(\pi(s), x)$, where $s \in S, x \in H^{A}, y \in H$.)

Proof. Let $\Phi$ be the isometry of $S \times H$ onto $R \times H$ given by the theorem of 6.1. Note that $\Phi(r, t, y)$ (where $(r, t) \in S \subset R \times I$ and $y \in H$ ) has first coordinate $r$; we may therefore write it as $\left(r, \Phi_{r}(t, y)\right)$ where $\Phi_{r}(t, y) \in H$. Define $\Theta$ by 


$$
\Theta(r, t, x, y)=\left(\psi(r, x), \Phi_{r}(t, y)\right)
$$

(where $r \in R, t \in S_{r}$-that is, $(r, t) \in S$-and $x \in H^{A}, y \in H$ ). Then $\Theta$ maps $S \times H^{A} \times H$ into $H^{A} \times H$, and it is easy to check that the above diagram commutes. To show that $\Theta$ is a pseudo-isometry (onto), consider four maps $\theta_{n}(n=1,2,3,4)$ defined as follows:

$\theta_{1}=(\psi \times i): R \times H^{A} \times H \rightarrow H^{A} \times H$, where $i$ is the identity map on $H$;

$\theta_{2}: R \times H \times H^{A} \rightarrow R \times H^{A} \times H$ interchanges the last two factors; that is $\theta_{2}(r, y, x)=(r, x, y)$;

$\theta_{3}=(\Phi \times j): S \times H \times H^{A} \rightarrow R \times H \times H^{A}$, where $j$ is the identity map of $H^{A}$;

$\theta_{4}: S \times H^{A} \times H \rightarrow S \times H \times H^{A}$ again interchanges the last two factors.

Note that $\theta_{3}(r, t, y, x)=\left(r, \Phi_{r}(t, y), x\right)$ where $t \in S_{r}$ (that is, $(r, t)$ $\in S), x \in H^{A}$ and $y \in H$.

It is easy to see that $\Theta=\theta_{1} \circ \theta_{2} \circ \theta_{3} \circ \theta_{4}$. Now clearly $\theta_{2}, \theta_{4}$ are isometries; so is $\theta_{3}$, as a product of two isometries, by (2). And $\theta_{1}$, as the product of a pseudo-isometry and an isometry, is a pseudo-isometry by (4). Hence, by 7.1 , the composition $\Theta$ is a pseudo-isometry, as required.

REMARK. As the proof shows, if $\psi$ here is actually an isometry, then so is $\Theta$.

8. The main theorem.

8.1. In this section we prove the main theorem of this paper:

THEOREM. Given a compact Hausdorff space $S$, say of weight $w$, and a completed regular Borel measure $\mu$ on $S$ such that $\mu(S)=1$, there exists a pseudo-isometry $\psi$ of the measure-theoretic product $(S, \mu) \times\left(I^{w}, m^{w}\right)$ onto $\left(I^{w}, m^{w}\right)$, where $m^{w}$ is the usual product measure derived from Lebesgue measure $m$ on $1 .\left({ }^{8}\right)$

What we shall actually prove is the equivalent assertion that there exists a pseudo-isometry $\psi$ of $(S, \mu) \times\left(H^{w}, M^{w}\right)$ onto $\left(H^{w}, M^{w}\right)$.

By standard topology, $S$ is homeomorphic to a subspace of the product space $I^{w}$. Thus we may (and do) assume $S \subset I^{w}$; of course the measure $\mu$ on $S$ has in general no relation to $\mathrm{m}^{w}$.

The presence of so many product spaces requires a fairly systematic notation. We regard $I^{w}$ as $I^{A}=\Pi\left\{I_{\alpha} \mid \alpha \in A\right\}$ where $A$ is an index set of

(8) For alternative topological hypotheses on $S$ under which the theorem is true, see the end of 9.1 below. 
cardinal $w$. For each $B \subset A$ we write $\pi_{B}$ for the projection map of $I^{A}$ onto $I^{B}$; that is, regarding $I^{A}$ as consisting of the functions from $A$ to $I$, as usual, $\pi_{B}$ restricts them to $B$. If $C \subset B \subset A$ we put $\pi_{C}^{B}=$ projection map from $I^{B}$ onto $I^{C}$. We write $\theta_{B}$ for $\pi_{B} \mid S, S_{B}$ for $\theta_{B}(S)=\pi_{B}(S), \theta_{C}^{B}$ for $\pi_{C}^{B} \mid S_{B}$. Note that $\theta_{C}=\theta_{C}^{B} \circ \theta_{B}$.

We write $\mu_{B}$ for the completed regular Borel measure induced on $S_{B}$ by projection; thus

$$
\begin{aligned}
& \mu_{B}(E)=\mu\left(\theta_{B}^{-1}(E)\right)=\mu\left(S \cap\left(E \times I^{A-B}\right)\right), \\
& \text { for each Borel (or measurable) set } E \subset S_{B} .
\end{aligned}
$$

It follows easily that:

If $C \subset B \subset A$, the inverse of the projection map $\theta_{C}^{B}:\left(S_{B}, \mu_{B}\right) \rightarrow\left(S_{C}, \mu_{C}\right)$ preserves measurability and measure.

Similarly we regard $H^{w}$ as $H^{A}$; we use $\omega_{B}, \omega_{C}^{B}$ to denote the projection maps $H^{A} \rightarrow H^{B}, H^{B} \rightarrow H^{C}$ (where $C \subset B \subset A$ ). As remarked in 7.3, the product measure $M^{B}$ on $H^{B}$ (where $M=m^{\aleph_{0}}$ ) is a complete regular Borel measure. Of course, the analogs of (1) and (2) hold for the measures $M^{B}$ and projection maps $\boldsymbol{\omega}_{C}^{B}$

8.2. Now let $P$ be the family of all ordered pairs $(B, \phi)$ where $\varnothing \neq$ $B \subset A$ and $\phi$ is a pseudo-isometry of $\left(S_{B}, \mu_{B}\right) \times\left(H^{B}, M^{B}\right)$ onto $\left(H^{B}, M^{B}\right)$. Define a partial order on $P$ by:

$(C, \psi) \leqslant(B, \phi)$ if and only if $C \subset B$ and the following diagram commutes:

$$
\begin{aligned}
\theta_{C}^{B} \times \omega_{C}^{B} S_{B} \times H^{B} \stackrel{\phi}{\longrightarrow} H^{B} \\
S_{C} \times H^{C} \stackrel{\psi}{\longrightarrow} H^{C}
\end{aligned}
$$

(Note that if $C=B$ and (3) holds then $\psi=\phi$.) One sees at once, on drawing the diagrams, that this does define a partial order (that is, is transitive).

We use Zorn's Lemma to deduce the existence of a maximal element of $P$. First we note

$$
P \neq \varnothing .
$$

For choose any $\alpha_{0} \in A$, and take $B=\left\{\alpha_{0}\right\}$; then $S_{B}$ is a compact subset of $I_{\alpha_{0}}=I$. The theorem of 5.1 gives a pseudo-isometry (in fact a Borel isometry) $\phi$ of $\left(S_{B}, \mu_{B}\right) \times H$ onto $H$; and since we can identify $H$ with $H^{B}$ here, we have $(B, \phi) \in P$. 
Next, let $L=\left\{\left(B_{\xi}, \phi_{\xi}\right) \mid \xi \in \Xi\right\}$ be a (nonempty) totally ordered subfamily of $P$; we construct an upper bound for $L$ in $P$. Put $B=\bigcup\left\{B_{\xi} \mid \xi \in \Xi\right\}$, and define $\phi: S_{B} \times H^{B} \rightarrow H^{B}$ as follows. Given $s \in S_{B}, x \in H^{B}$ and $\beta \in B$, there exists $\xi \in \Xi$ such that $\beta \in B_{\xi}$; choose any such $\xi$, and put $y_{\beta}=\beta$ th coordinate of the point $\phi_{\xi}\left(\pi_{B_{\xi}}^{B}(s), \omega_{B_{\xi}}^{B}(x)\right) \in H^{B}$. Then $y_{\beta}$ is independent of the choice of $\xi$; for if also $\beta \in B_{\eta}$ where $\eta \in \Xi$ we may assume without loss of generality that $\left(B_{\xi}, \phi_{\xi}\right) \leqslant\left(B_{\eta}, \phi_{\eta}\right)$; and the assertion follows on applying the commutativity of the diagram in (3).

We define $\phi(s, x)$ to be the point of $H^{B}$ for which the $\beta$ th coordinate is $y_{\beta}(\beta \in B)$. Because $y$ is independent of the choice of $\xi$ above, we readily verify that

$$
\omega_{B_{\xi}}^{B}(\phi(s, x))=\phi_{\xi}\left(\pi_{B_{\xi}}^{B}(s), \omega_{B_{\xi}}^{B}(x)\right) \text { for all } \xi \in \Xi, s \in S_{B}, x \in H^{B} .
$$

Thus, once it is shown that $\phi$ is a pseudo-isometry of $S_{B} \times H^{B}$ onto $H^{B}$, it will follow that $(B, \phi) \in P$ and that $\left(B_{\xi}, \phi_{\xi}\right) \leqslant(B, \phi)$ for all $\xi \in \Xi$, as required. To verify that $\phi$ is a pseudo-isometry as stated, we first check that $\phi$ is surjective. Given $y \in H^{B}$, there is for each $\xi \in \Xi$ a unique $\left(s_{\xi}, x_{\xi}\right) \in$ $S_{B} \times H^{B}$ such that $\phi_{\xi}\left(s_{\xi}, x_{\xi}\right)=\omega_{B_{\xi}}^{B}(y)$. If $\eta \in \Xi$ is such that $\left(B_{\xi}, \phi_{\xi}\right) \leqslant$ $\left(B_{\eta}, \phi_{\eta}\right)$ we see from (3) that $s_{\xi}$ and $s_{\eta}$ have the same $\beta$ th coordinate for each $\beta \in B_{\xi}$, and similarly for $x_{\xi}$ and $x_{\eta}$. We thus obtain well-defined points $s \in S_{B}, x \in H^{B}$, such that $\theta_{B_{\xi}}^{B}(s)=s_{\xi}$ and $\omega_{B_{\xi}}^{B}(x)=x_{\xi}$ for all $\xi \in \Xi$. It is now easy to see that $\phi(s, x)=y$.

Similarly it is a straightforward matter to check that $\phi$ is injective.

Next we show:

$$
\begin{aligned}
& \text { If } E \in H^{B} \text { is measurable, then so is } \phi^{-1}(E) \subset S_{B} \times H^{B} \text {, } \\
& \text { and }\left(\mu_{B} \times M^{B}\right)\left(\phi^{-1}(E)\right)=M^{B}(E) \text {. }
\end{aligned}
$$

First suppose $E$ is a Borel "cylinder set" on a finite number of coordinates; that is, $E=\left(\omega_{C}^{B}\right)^{-1}\left(E_{C}\right)=E_{C} \times H^{B-C}$ where $C$ is a finite (nonempty) subset of $B$, and where $E_{C}$ is a Borel subset of $H^{C}$. Each $\beta \in C$ is in some $B_{\xi}$; and the resulting finite set of members $\left(B_{\xi}, \phi_{\xi}\right)$ of $L$ has a greatest member, say $\left(B_{\eta}, \phi_{\eta}\right)$. Thus $C \subset B_{\eta}$, and $E$ is of the form $\left(\omega_{B_{\eta}}^{B}\right)^{-1}\left(E_{\eta}\right)$ where $E_{\eta}$ is a Borel subset of $H^{B}$. Hence $\left(\phi_{\eta}\right)^{-1}\left(E_{\eta}\right)=F_{\eta}$ say, is a measurable subset of $S_{B_{\eta}} \times H^{B} \eta$, with

$$
\left(\mu_{B_{\eta}} \times M^{B} \eta\right)\left(F_{\eta}\right)=M^{B} \eta\left(E_{\eta}\right)=M^{B}(E) .
$$

Now it is easy to see that $\phi^{-1}(E)=\left(\theta_{B_{\eta}}^{B} \times \omega_{B_{\eta}}^{B}\right)^{-1}\left(F_{\eta}\right)$. From (2), $\left(\theta_{B_{\eta}}^{B}\right)^{-1}$ preserves measurability and measure, and the same is true of $\left(\omega_{B_{\eta}}^{B}\right)^{-1}$. Thus (cf. 7(1)) the same is also true of $\left(\theta_{B_{\eta}}^{B} \times \omega_{B_{\eta}}^{B}\right)^{-1}$; which shows that $\phi^{-1}(E)$ 
is measurable and that $\left(\mu_{B} \times M^{B}\right)\left(\phi^{-1}(E)\right)=\left(\mu_{B_{\eta}} \times M^{B} \eta\right) F_{\eta}=M^{B}(E)$.

The family of all measurable sets $E \subset H^{B}$ for which (6) is true, being a $\sigma$-field (from 4.4), thus contains the Baire sets in $H^{B}$. But every $M^{B}$-null set is contained in a null Baire set, so that (6) is also true for null sets, and thus holds unrestrictedly.

Next we show:

Given a measurable set $D \subset S_{B} \times H^{B}$, there exists a measurable set $D^{\prime} \subset S_{B} \times H^{B}$, differing from $D$ by a null set, such that $\phi\left(D^{\prime}\right)$ is measurable (in $\left(H^{B}, M^{B}\right)$ ).

Again we first show this when $D$ is a Borel cylinder set on a finite number of coordinates. More precisely (recalling that $S_{B} \subset I^{B}$ ) we require $D$ to be of the form $\left(\theta_{C_{1}}^{B}\right)^{-1}\left(D_{1}\right) \times\left(\omega_{C_{2}}^{B}\right)^{-1}\left(D_{2}\right)$, where $C_{1}, C_{2}$ are finite subsets of $B$, and where $D_{1}, D_{2}$ are Borel subsets of $H^{C 1}, H^{C 2}$ respectively. As before, we have $C_{1} \cup C_{2} \subset B_{\xi}$ for some $\xi \in \Xi$, and $D$ can be written as

$$
D=\left(\theta_{B_{\xi}}^{B}\right)^{-1}(E) \times\left(\omega_{B_{\xi}}^{B}\right)^{-1}(F)
$$

where $E, F$ are Borel subsets of $S_{B_{\xi}}, H^{B} \xi$, respectively. Because $\phi_{\xi}$ is a pseudo-isometry, there exists a measurable set $G$ in $S_{B_{\xi}} \times H^{B_{\xi}}$ such that $G$ differs from $E \times F$ by a $\left(\mu_{B_{\xi}} \times M^{B_{\xi}}\right.$ )-null set and $\phi_{\xi}(G)$ is measurable (in $\left.\left(H^{B_{\xi}}, M^{B}\right)\right)$ ). Now define $D^{\prime}=\left(\theta_{B_{\xi}}^{B} \times \omega_{B_{\xi}}^{B}\right)^{-1}(G)$; it follows as before (in proving (6)) that $D^{\prime}$ is measurable and differs from $D$ by a null set. A routine calculation shows that $\phi\left(D^{\prime}\right)=\phi_{\xi}(G) \times H^{B-B} \xi$, which is measurable. Thus (7) holds for $D$ of this special form. Hence it holds for the $\sigma$-field generated by these sets; that is, for the Baire sets in $S_{B} \times H^{B}$. But, by a standard property of regular Borel measures, every measure class in $S_{B} \times H^{B}$ contains a Baire set, so (7) follows in full generality.

This proves that $\phi$ is indeed a pseudo-isometry from $S_{B} \times H^{B}$ onto $H^{B}$, and thus that $(B, \phi)$ is an upper bound for $L$ in $P$.

8.3. By Zorn's Lemma, $P$ therefore has a maximal element, say $(C, \psi)$. We now show that $C=A$, thus establishing that $\psi$ is a pseudo-isometry of $S \times H^{w}$ onto $H^{w}$ as required.

Suppose $\alpha \in A-C$; put $B=C \cup\{\alpha\}, S^{\prime}=\pi_{B}(S)=S_{B}, R=\pi_{C}(S)=$ $S_{C}$. We apply the theorem of 7.3 to the pseudo-isometry $\psi: R \times H^{C} \rightarrow H^{C}$. Note that the hypotheses of that theorem apply here: $R$ is a compact Hausdorff space, $S^{\prime}$ is a closed subset of $R \times I_{\alpha}$, projecting (under $\pi_{C}^{B}$ ) onto all of $R$; and, because of (2) above, the measures $\mu_{C}$ on $S^{\prime}$ and $\mu_{B}$ on $R$ are appropriately related. We therefore obtain a pseudo-isometry $\Theta$ of 
$S^{\prime} \times H^{C} \times H_{\alpha}$ onto $H^{C} \times H_{\alpha}$-that is, of $S_{B} \times H^{B}$ onto $H^{B}$-making the diagram

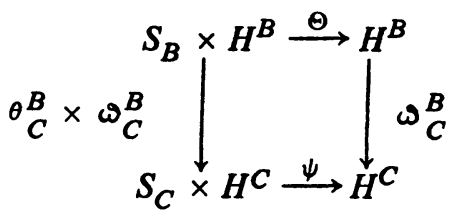

commute. Thus $(B, \Theta) \in P$, and $(C, \psi)$ is strictly $<(B, \Theta)$. This contradicts the maximality of $(C, \psi)$ and establishes the theorem

9. A Baire isomorphism theorem.

9.1. The theorem of 8.1 gives a pseudo-isometry $\psi$; as pointed out earlier, $\psi$ can be regarded as an isometry if one replaces the $\sigma$-field of measurable sets in $S$ by a suitable (full) $\sigma$-subfield. There is a certain lack of precision here; it would be interesting to know whether $\psi$ can be chosen so as to be an isometry (without this replacement). Even more, can $\psi$ be made to be a Borel isometry? While these questions remain unanswered, we are able to show that, under rather different topological assumptions about $S$, it can be arranged that $\psi$ is a Baire isometry-that is, both $\psi$ and $\psi^{-1}$ take Baire sets to Baire sets of the same measure. The theorem is as follows.

THEOREM. Suppose $S$ is Baire isomorphic to a product $I^{w}$ of unit intervals, and that $\mu$ is a Baire measure on $S$ with $\mu(S)=1 .\left(^{9}\right)$ Then there exists a Baire isometry $\psi$ of $(S, \mu) \times\left(I^{w}, m^{w}\right)$ onto $\left(I^{w}, m^{w}\right)$.

As a corollary, the theorem applies if $S$ is topologically a Baire set (not necessarily compact) in $I^{w}, w \geqslant 1$. For if $w \leqslant \aleph_{0}, S$ is then a separable absolute Borel set, and is therefore Borel (= Baire) isomorphic to $I$ (unless $S$ is countable, in which case the conclusion of the theorem holds trivially). And if $w>\aleph_{0}, S$ is homeomorphic to $S^{\prime} \times I^{w}$ where $S^{\prime}$ is a separable absolute Borel set, so that $S^{\prime} \times I$ is Baire isomorphic to $I$ and $S$ is Baire isomorphic to $I^{w}$.

The theorem also applies, for example, when $S$ is topologically the product of $\boldsymbol{w}$ separable absolute Borel sets-open intervals or Cantor sets, for instance.

Of course, in these examples (as in the theorem) the measure $\mu$ is to be a Baire measure. We could instead suppose $\mu$ to be extended to a regular Borel measure in the above theorem; then $\psi$ becomes a pseudo-isometry, and we obtain a variant of the theorem of 8.1 under alternative topological hypotheses on $S$.

(9) A "Baire isomorphism" is a bijection preserving Baire sets both ways. 
9.2. In proving the above theorem, we may assume that $S=I^{w}$ (though the measure $\mu$ is of course unrelated to $m^{w}$ ). The proof now consists in going over the entire argument leading to the theorem of 8.1 , in the case $S=I^{w}$, and checking that with suitable modifications it now ensures that Baire sets behave properly. The point is that, because now $S=I^{w}$, the spaces $S_{B}$ arising in the argument of $\S 8$ will themselves be products of intervals; thus the earlier results will be needed only for products of intervals, and the resulting topological simplifications will enable us to deal with the Baire sets. We now describe the necessary modifications in more detail, reverting to the notation previously used (so that the symbol $S$ will temporarily lose the meaning given to it above).

The results of $\S 3$, which were previously used to guarantee Borel measurability in the later constructions, will now be used to guarantee Baire measurability; no change in $\S 3$ is needed for this. We shall need the disintegration theorem of $\S 4$ only when $S=R \times I$, and in this case (or more generally if $S$ is a compact Baire subset of $R \times I$ ) we replace the word "Borel", applied to sets, measures and measurability, by the word "Baire" throughout $\$ 4$ (note that a finite Baire measure is automatically regular). With this replacement, the argument in $\$ 4$ needs only two slight modifications. First, in the proof of equation (2) (in 4.2), we must now ensure that $\pi(F)$ is a Baire (rather than merely Borel) set. To do this, note that $F$ can now be taken to be a countable union of zero-sets, and that $\pi$ takes zero-sets to zero-sets (as one can show via the fact that zero-sets coincide with closed $G_{\delta}$ sets). Second, in proving (a) in 4.3, we need to know that $\left\{r \in R \mid a_{r} \in F\right\}$ is a Baire set; this is now trivial, because now $a_{r}=0$ for all $r \in R$.

In the situation dealt with in $\S 5$ the notions "Baire" and "Borel" are synonymous, so no alteration is needed. We shall need the result of $\S 6$ only when $S=R \times I$; in this case, it was shown in 6.8 that the isomorphism $\Phi$ (of 6.1) can be taken to be a Borel isometry. By replacing "Borel" throughout the proof by "Baire", we obtain a proof of the corresponding theorem giving a Baire isometry in 6.1 (when $S=R \times I$ and $\mu$ is a Baire measure on $S$ ).

The extension theorem of 7.3 will likewise be needed only when $S=R \times I$; and in that case we need to know that, if $\psi$ is a Baire isometry, then $\Theta$ can be taken to be a Baire isometry too. This is shown by the proof in 7.3, which (granted that $S=R \times I$ ) needs no alteration.

Now, assuming $S=I^{w}$, we turn to the argument in $\S 8$, of course replacing the Borel measures by Baire measures throughout. Note that now $S_{B}=$ $I^{B}$ and $\theta_{C}^{B}=\pi_{C}^{B}$, in the notation of 8.1. The family $P(8.2)$ will now consist of all ordered pairs $(B, \phi)$ where $\varnothing \neq B \subset A$ and $\phi$ is a Baire isometry of $\left(S_{B}, \mu_{B}\right) \times\left(H^{B}, M^{B}\right)$ onto $\left(H^{B}, M^{B}\right)$. The partial ordering of $P$ will be the same as before (8(3)). The proof, in 8.2 , that Zorn's Lemma is applicable is 
unchanged, except that it must now be shown that $\phi$ is a Baire isometry; in other words, $8(6)$ must be replaced by:

$$
\begin{aligned}
& \text { If } E \subset H^{B} \text { is a Baire set, then so is } \phi^{-1}(E) \subset S_{B} \times H^{B} \\
& \left(=I^{B} \times H^{B}\right) ; \text { and }\left(\mu_{B} \times M^{B}\right)\left(\phi^{-1}(E)\right)=M^{B}(E)
\end{aligned}
$$

and $8(7)$ is to be replaced by:

$$
\text { If } D \text { is a Baire set in } S_{B} \times H^{B} \text {, then } \phi(D) \text { is a }
$$

Baire set in $H^{B}$.

Now ( $\left.6^{\prime}\right)$ follows by essentially the same argument as that used to prove (6) in 8.2 (the argument is now even a little simpler, as only Baire sets have to be considered). And the proof of $\left(7^{\prime}\right)$ is similar to that of $\left(6^{\prime}\right)$ (and simpler than that of (7) in 8.2).

Thus, as before, $P$ has a maximal element $(C, \psi)$; and it follows that $C=A$ by substantially the same reasoning as in 8.3 ; note that we need the Baire analog of the theorem of 7.3 here only in the case $S^{\prime}=R \times I_{\alpha}$, for which it has been shown above to be valid. This completes the proof of the present theorem.

10. An isomorphism theorem for maps.

10.1. We conclude the paper by deducing from the previous work a theorem on "smoothing" continuous maps with measure-preserving inverses; roughly speaking, while such a map need not be isomorphic to a projection (from a product of intervals to a subproduct), its product with a suitable projection is isomorphic to a projection. The resulting theorem includes the theorem of 8.1 , and may be regarded as a generalization of the extension theorem of 7.3

THEOREM. Suppose $R, S$ are compact Hausdorff spaces, and $\theta$ is a continuous map of $S$ onto $R$. Let $\mu$ be a complete regular Borel measure on $S$, with $\mu(S)=1$, and let $\lambda$ be the (complete regular Borel) measure on $R$ induced by $\theta$ (that is, $\lambda=\mu \circ \theta^{-1}$ ). Then there exist index sets $A, B$, with the following properties: (i) $\varnothing \neq B \subset A$; (ii) there exists a pseudo-isometry $\phi$ of $\left(R \times I^{B}, \lambda \times m^{B}\right)$ onto $\left(I^{B}, m^{B}\right)$; (iii) for every such $\phi$ there exists a pseudo-isometry $\psi$ of $\left(S \times I^{A}, \mu \times m^{A}\right)$ onto $\left(I^{A}, m^{A}\right)$ such that the following diagram commutes, where $\pi$ denotes the projection map of $I^{A}$ onto $I^{B}$ :

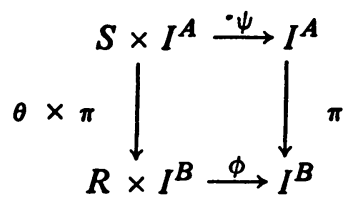


10.2. We first prove the above theorem in a special case. Suppose $R \subset I^{B}$ and $S \subset I^{A}$, where $B$ is an arbitrary infinite subset of an arbitrary infinite set $A$; and suppose $\theta=\pi \mid S$, where $\pi$ is the projection map from $I^{A}$ to $I^{B}$. By the theorem of 8.1 applied to $R$, we get a pseudo-isometry $\phi$ of the measuretheoretic product $(R \times \lambda) \times\left(I^{w}, m^{w}\right)$ onto $\left(I^{w}, m^{w}\right)$ where $w$ is the weight of $R$. Trivially $w$ can be replaced by any larger cardinal; thus we may replace $\left(I^{w}, m^{w}\right)$ throughout by $\left(I^{B}, m^{B}\right)$, or equivalently by $\left(H^{B}, M^{B}\right)$. Referring to the argument in 8.2 , we then have $(B, \phi) \in P$, and by Zorn's Lemma there is therefore, for any such $\phi$, a maximal element $(C, \psi)$ of $P$ such that $(B, \phi) \leqslant$ $(C, \psi)$. But the argument in 8.3 shows as before that $C=A$. Thus $\psi$ is a pseudo-isometry making the diagram

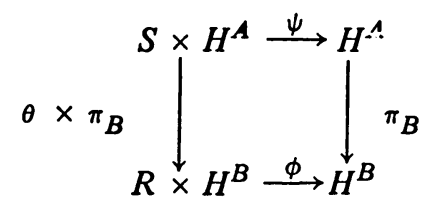

commute. Since $H^{A}$ and $H^{B}$ are the "same" as $I^{A}$ and $I^{B}$, this proves the theorem in this case.

10.3 In the general case, we first define suitable sets $A, B$. Take $A$ to be the set of all continuous maps $a: S \rightarrow I, C$ to be the set of all continuous maps $c: R \rightarrow I$, and $B$ to consist of those continuous maps $c: S \rightarrow I$ that are expressible as $b=c \circ \theta$, where $c \in C$. Trivially $B \subset A$ and $B$ is infinite. There is a natural bijection $\theta^{*}$ of $C$ onto $B$, given by $\theta^{*}(c)=c \circ \theta$, and $\theta^{*}$ induces a homeomorphism $\xi$ of $I^{C}$ onto $I^{B}$ (sending the point $x: C \rightarrow I$ of $I^{C}$ to the point $y: B \rightarrow I$ of $I^{B}$ for which $\left.y(b)=x\left(\theta^{*-1}(b)\right), b \in B\right)$.

Let $e$ be the evaluation map of $R$ into $I^{C}$; that is, for each $r \in R$, $e(r)$ is the point $x: C \rightarrow I$ of $I^{C}$ for which $x(c)=c(r), c \in C$. It is well known that $e$ is a homeomorphism. Similarly the evaluation map $e^{\prime}$ of $S$ into $I^{A}$ is a homeomorphism. Write

$$
S_{1}=e^{\prime}(S) \subset I^{A}, \quad R_{1}=\xi(e(R)) \subset I^{B}, \quad \theta_{1}=\pi \mid S_{1},
$$

where $\pi$ is the projection map of $I^{A}$ onto $I^{B}$. Observe that the diagram

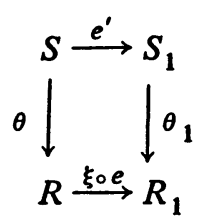

commutes, and that all maps in it are surjective. The maps $e^{\prime}, \xi \circ e$ are homeomorphisms, and we use them to transfer the measures $\mu$ on $S$ and $\lambda$ on $R$ to measures $\mu_{1}$ on $S_{1}$ and $\lambda_{1}$ on $R_{1}$; that is, $\mu_{1}(E)=\mu\left(e^{\prime-1} E\right)$ for each 
Borel set $E \subset S_{1}$, and similarly for $\lambda_{1}$. Using the commutativity of the above diagram, we see that $\lambda_{1}$ is precisely the measure $\mu \circ \theta_{1}^{-1}$ induced on $R_{1}$ by $\theta_{1}$.

Again, on writing $i, j$ for the respective identity maps on $I^{A}, I^{B}$, we see from the commutativity of the above diagram that the diagram

$$
\theta \times\left.\pi\right|_{R \times I^{B} \stackrel{(\xi \circ e) \times J}{\longrightarrow}} ^{S \times I^{A} \stackrel{e^{\prime} \times i}{\longrightarrow} S_{1}} \underbrace{\theta_{1} \times \pi}_{I^{B}}
$$

also commutes.

Now the special case considered in 10.2 applies to the measure spaces $\left(R_{1}, \lambda_{1}\right),\left(S_{1}, \mu_{1}\right)$ and the map $\theta_{1}$; so we get the existence of pseudo-isometries $\phi_{1}, \psi_{1}$ making the diagram

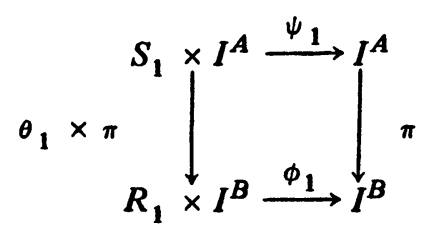

commute. Combining this with the previous diagram, and defining $\psi=$ $\psi_{1} \circ\left(e^{\prime} \times i\right), \phi=\phi_{1} \circ((\xi \circ e) \times j)$, we have the commuting diagram required by the theorem, once it is verified that $\psi$ and $\phi$ are pseudo-isometries. But $e^{\prime} \times i$ and $(\xi \circ e) \times j$ are isometries, from the way in which $\mu_{1}$ and $\lambda_{1}$ were defined; so the result follows from 7.1.

All that remains is to note that every pseudo-isometry $\phi$, of $\left(R \times I^{B}\right.$, $\left.\lambda \times m^{B}\right)$ onto $\left(I^{B}, m^{B}\right)$, can arise here, since we have only to take $\phi_{1}=$ $\phi \circ((\xi \circ e) \times j)^{-1}$, which is a pseudo-isometry of $R_{1} \times I^{B}$ onto $I^{B}$ because of 7.1 again.

10.4. It would be interesting to know whether a satisfactory Baire analog of this theorem is valid. The following very special result can be obtained by a straightforward adaptation of the argument in 10.2: Suppose $R=I^{B}$ and $S=I^{A}$ (where $B \subset A$ and is infinite), and that $\theta=\pi$, the projection map from $I^{A}$ to $I^{B}$. Let $\mu$ be a Baire measure on $S$, with $\mu(S)=1$, and let $\lambda=\mu \circ \theta^{-1}$. Then there exist Baire isometries $\phi$ of $\left(R \times I^{B}, \lambda \times m^{B}\right)$ onto $\left(I^{B}, m^{B}\right)$ and $\psi$ of $\left(S \times I^{A}, \mu \times m^{A}\right)$ onto $\left(I^{A}, m^{A}\right)$, making the diagram

$$
\begin{array}{ll}
S \times I^{A} \stackrel{\psi}{\longrightarrow} I^{A} \\
\theta \times \pi \downarrow \\
R \times I^{B} \stackrel{\phi}{\longrightarrow} I^{B}
\end{array}
$$

commute; moreover, for every such $\phi$, a corresponding $\psi$ exists. 
The hypotheses here would have to be considerably relaxed before the method of 10.3 could be applied to generalize the result further.

ADDED IN PROOF. The proof of the disintegration theorem of $\S 4$ can be considerably shortened. After defining $f(H, r)\left(\right.$ p. 12), put $u_{x}(r)=$ $\inf \{f(R \cap[0, t]) \mid t$ rational, $1 \geqslant t \geqslant x\} \quad(x \in I, r \in R)$. One can take $\mu_{r}$, for $r \in R-N^{\prime \prime}$ (where $N^{\prime \prime}$ is a suitable null set), to be the Lebesgue-Stieltjes measure on $I$ defined by $u_{x}(r)$, and verify that this works. (On $N^{\prime \prime}, \mu_{r}$ is defined as before, p. 15.)

This theorem can also be deduced from a general disintegration theorem due to M. Valadier (Désintegration d'une mesure sur un produit, C. R. Acad. Sci. Paris 276 (1973), 33-35). Other, independent proofs of the general theorem have been given by S. Graf and by the author. I am grateful to Dr. Graf for calling my attention to Valadier's proof, and for showing me his own.

\section{REFERENCES}

1. P. R. Halmos, Measure theory, Van Nostrand, Princeton, N. J., 1950. MR 11, 504.

2. K. Kuratowski, Topology. Vol. 1, Academic Press, New York; PWN, Warsaw, 1966. MR 36 \#840.

3. D. Maharam, On homogeneous measure algebras, Proc. Nat. Acad. Sci. U.S.A. 28 (1942), 108-111. MR 4, 12.

4. - Decompositions of measure algebras and spaces, Trans. Amer. Math. Soc. 69 (1950), 142-160. MR 12, 167.

5. - Automorphisms of products of measure spaces, Proc. Amer. Math. Soc. 9 (1958), 702-707. MR 20 \#3963.

6. - On a theorem of von Neumann, Proc. Amer. Math. Soc. 9 (1958), 987994. MR $21 \# 4220$.

7. R. Panzone and C. Segovia, Measurable transformations on compact spaces and orthonormal systems on compact groups, Rev. Un. Mat. Argentina 22 (1964), 83-102. MR 30 \#2119.

8. A. Ionescu Tulcea and C. Ionescu Tulcea, Topics in the theory of lifting, Ergebnisse der Mathematik und ihrer Grenzgebiete, Band 48, Springer-Verlag, New York, 1969. MR 43 $\# 2185$.

9. C. Ionescu Tulcea, Sur certains endomorphismes de $L_{c}^{\infty}(Z, \mu)$, C. R. Acad. Sci. Paris 261 (1965), $4961-4963$. MR 33 \#4699.

10. H. D. Ursell, Some methods of proving measurability, Fund. Math. 32 (1939), 311330.

11. J. von Neumann, Einige Satze über messbare Abbildungen, Ann. of Math. 33 (1932), 574-586.

DEPARTMENT OF MATHEMATICS, UNIVERSITY OF ROCHESTER, ROCHESTER, NEW YORK 14627 